\title{
ITEM DR1
}
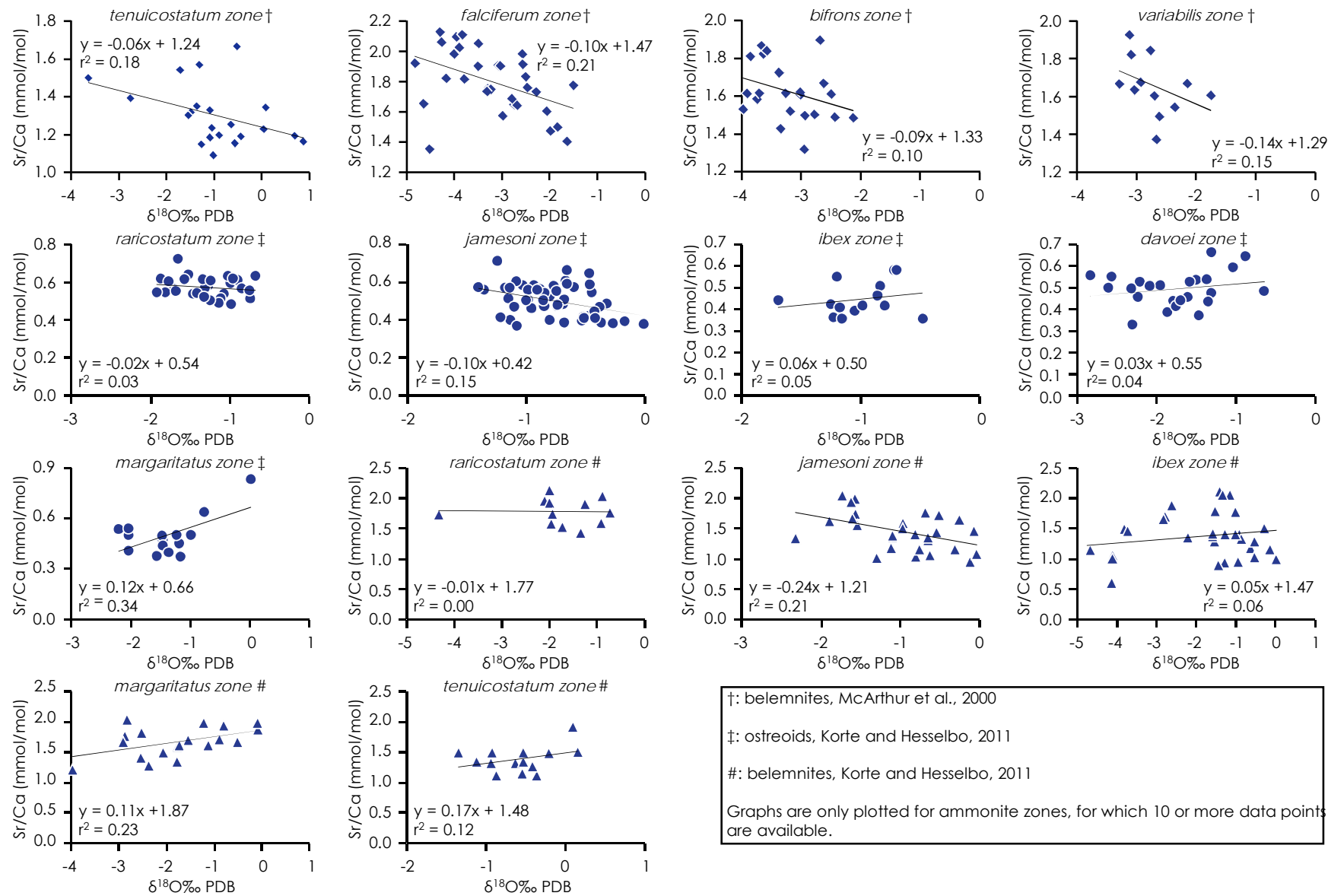

Figure DR1: cross plots of $\delta^{18} \mathrm{O}$ values and $\mathrm{Sr} / \mathrm{Ca}$ ratios of belemnites and ostreoids from different ammonite zones. Correlations are generally weak and slopes of correlations ambiguous. 

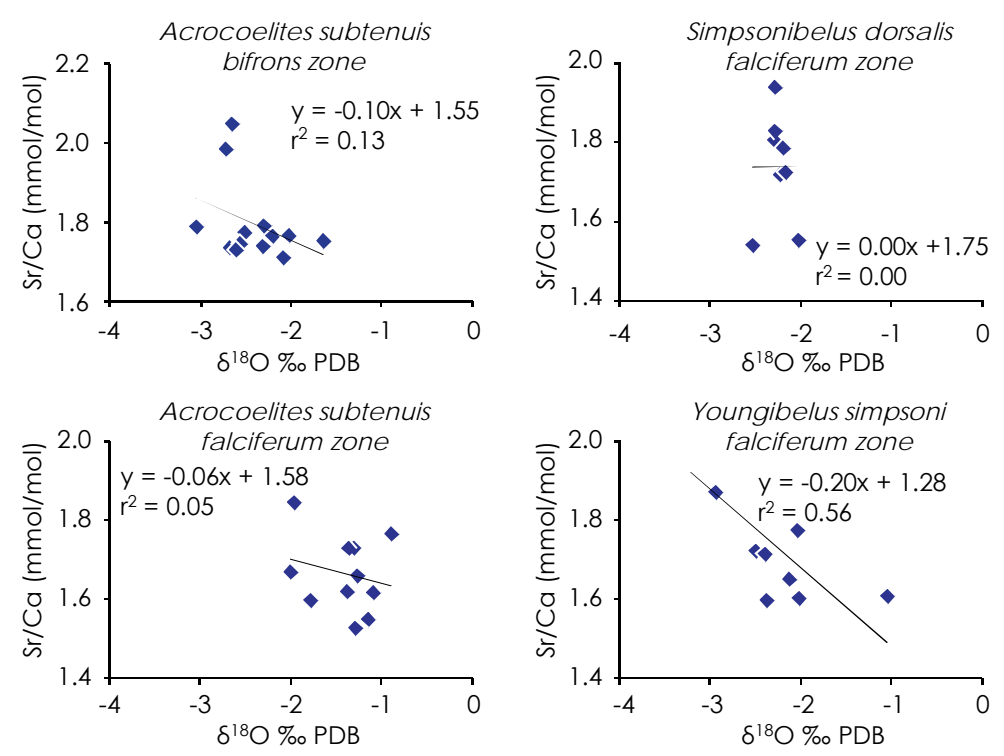

Figure DR2: Cross plots of $\delta^{18} \mathrm{O}$ values and $\mathrm{Sr} / \mathrm{Ca}$ ratios of single species of belemnites from bifrons and falciferum ammonite zones. Apart from a single case, correlations are weak and slopes of correlation are $\leq-0.20(\mathrm{mmol} / \mathrm{mol}) / \%$. Data are from Li et al., 2012. 
Table DR1: Locations of Sections used for this compilation.

\begin{tabular}{lcc}
\hline name & Latitude & Longitude \\
\hline Hawsker Bottoms & $54^{\circ} 27^{\prime} 24^{\prime \prime} \mathrm{N}$ & $0^{\circ} 32^{\prime} 20^{\prime \prime} \mathrm{W}$ \\
Kettleness & $54^{\circ} 32^{\prime} 03^{\prime \prime} \mathrm{N}$ & $0^{\circ} 42^{\prime} 58^{\prime \prime} \mathrm{W}$ \\
Peak & $54^{\circ} 24^{\prime} 23^{\prime \prime} \mathrm{N}$ & $0^{\circ} 29^{\prime} 25^{\prime \prime} \mathrm{W}$ \\
Port Mulgrave & $54^{\circ} 32^{\prime} 50^{\prime \prime} \mathrm{N}$ & $0^{\circ} 46^{\prime} 01^{\prime \prime} \mathrm{W}$ \\
Ravenscar & $54^{\circ} 24^{\prime} 23^{\prime \prime} \mathrm{N}$ & $0^{\circ} 29^{\prime} 25^{\prime \prime} \mathrm{W}$ \\
Robin Hood's Bay & $54^{\circ} 24^{\prime} 53^{\prime \prime} \mathrm{N}$ & $0^{\circ} 30^{\prime} 58^{\prime \prime} \mathrm{W}$ \\
Runswick Bay & $54^{\circ} 32^{\prime} 03^{\prime \prime} \mathrm{N}$ & $0^{\circ} 44^{\prime} 54^{\prime \prime} \mathrm{W}$ \\
Saltwick Nab & $54^{\circ} 29^{\prime} 21^{\prime \prime} \mathrm{N}$ & $0^{\circ} 35^{\prime} 18^{\prime \prime} \mathrm{W}$ \\
Staithes & $54^{\circ} 33^{\prime} 29^{\prime \prime} \mathrm{N}$ & $0^{\circ} 46^{\prime} 47^{\prime \prime} \mathrm{W}$ \\
Ardnish & $57^{\circ} 15^{\prime} 13^{\prime \prime} \mathrm{N}$ & $5^{\circ} 50^{\prime} 39^{\prime \prime} \mathrm{W}$ \\
Borreraig & $57^{\circ} 10^{\prime} 30^{\prime \prime} \mathrm{N}$ & $5^{\circ} 56^{\prime} 15^{\prime \prime} \mathrm{W}$ \\
Bearreraig & $57^{\circ} 29^{\prime} 49^{\prime \prime} \mathrm{N}$ & $6^{\circ} 08^{\prime} 40^{\prime \prime} \mathrm{W}$ \\
Hallaig Waterfall & $57^{\circ} 22^{\prime} 30^{\prime \prime} \mathrm{N}$ & $6^{\circ} 00^{\prime} 13^{\prime \prime} \mathrm{W}$ \\
Lochaline & $56^{\circ} 32^{\prime} 36^{\prime \prime} \mathrm{N}$ & $5^{\circ} 45^{\prime} 13^{\prime \prime} \mathrm{W}$ \\
\hline Alina & $50^{\circ} 47^{\prime} 03^{\prime \prime} \mathrm{N}$ & $1^{\circ} 02^{\prime} 04^{\prime \prime} \mathrm{E}$ \\
Sowa-Gliński & $50^{\circ} 47^{\prime} 05^{\prime \prime} \mathrm{N}$ & $1^{\circ} 03^{\prime} 00^{\prime \prime} \mathrm{E}$ \\
Gnaszyn & $50^{\circ} 48^{\prime} 03^{\prime \prime} \mathrm{N}$ & $19^{\circ} 02^{\prime} 41^{\prime \prime} \mathrm{E}$ \\
Kawodrza & $50^{\circ} 48^{\prime} 09^{\prime \prime} \mathrm{N}$ & $1^{\circ} 033^{\prime} 50^{\prime \prime} \mathrm{E}$ \\
Faustianka & $51^{\circ} 02^{\prime} 35^{\prime \prime} \mathrm{N}$ & $1^{\circ} 32^{\prime} 39^{\prime \prime} \mathrm{E}$ \\
Pacanów & $50^{\circ} 54^{\prime} 25^{\prime \prime} \mathrm{N}$ & $18^{\circ} 43^{\prime} 27^{\prime \prime} \mathrm{E}$ \\
Młynka & $50^{\circ} 06^{\prime} 31^{\prime \prime} \mathrm{N}$ & $1^{\circ} 41^{\prime} 17^{\prime \prime} \mathrm{E}$ \\
Wieluń & $51^{\circ} 12^{\prime} 43^{\prime \prime} \mathrm{N}$ & $18^{\circ} 34^{\prime} 22^{\prime \prime} \mathrm{E}$ \\
Stare Gliny & $50^{\circ} 21^{\prime} 00^{\prime \prime} \mathrm{N}$ & $1^{\circ} 35^{\prime} 18^{\prime \prime} \mathrm{E}$ \\
Ogrodzieniec & $50^{\circ} 27^{\prime} 34^{\prime \prime} \mathrm{N}$ & $1^{\circ} 30^{\prime} 31^{\prime \prime} \mathrm{E}$ \\
\hline & &
\end{tabular}




\section{References}

Korte, C., and Hesselbo, S.P., 2011, Shallow marine carbon andoxygen isotope and elemental records indicate icehouse-greenhouse cycles during the Early Jurassic: Paleoceanography, v. 26, PA4219, doi:10.1029/2011PA002160.

McArthur, J.M., Doyle, P., Leng, M.J., Reeves, K., Williams, C.T., Garcia-Sanchez, R., Howarth, R.J., 2007. Testing palaeo-environmental proxies in Jurassic belemnites: $\mathrm{Mg} / \mathrm{Ca}, \mathrm{Sr} / \mathrm{Ca}, \mathrm{Na} / \mathrm{Ca}, \delta^{18} \mathrm{O}$ and $\delta^{13} \mathrm{C}$. Palaeogeography, Palaeoclimatology, Palaeoecology 252, 464-480.

Li, Q., McArthur, J.M., Atkinson, T.C., 2012. Lower Jurassic belemnites as indicators of palaeotemperature. Palaeogeography, Palaeoclimatology, Palaeoecology 315-316, 38-45. 


\section{ITEM DR2}

Belemnite Data

\begin{tabular}{|c|c|c|c|c|c|}
\hline section & sample No & $\begin{array}{c}\text { Zone } \\
\text { No. }\end{array}$ & $\begin{array}{c}\mathrm{Sr} / \mathrm{Ca} \\
\mathrm{mmol} / \mathrm{mol}\end{array}$ & $\begin{array}{c}\mathrm{Mn} / \mathrm{Ca} \\
\mathrm{mmol} / \mathrm{mol}\end{array}$ & source \\
\hline & 230 & 47.75 & 1.24 & 0.03 & Wierzbowski et al., 2012 \\
\hline & 232 & 47.75 & 1.23 & 0.02 & Wierzbowski et al., 2012 \\
\hline & 233 & 47.75 & 1.21 & 0.04 & Wierzbowski et al., 2012 \\
\hline & 285 & 47.75 & 1.27 & 0.05 & Wierzbowski et al., 2012 \\
\hline & 286 & 47.75 & 1.18 & 0.05 & Wierzbowski et al., 2012 \\
\hline & 266 & 47.25 & 1.17 & 0.02 & Wierzbowski et al., 2012 \\
\hline & 267 & 47.25 & 1.25 & 0.02 & Wierzbowski et al., 2012 \\
\hline & 268 & 47.25 & 1.16 & 0.01 & Wierzbowski et al., 2012 \\
\hline & 269 & 47.25 & 1.22 & 0.01 & Wierzbowski et al., 2012 \\
\hline & 225 & 46.75 & 1.16 & 0.05 & Wierzbowski et al., 2012 \\
\hline & 226 & 46.75 & 1.13 & 0.02 & Wierzbowski et al., 2012 \\
\hline & 236 & 46.75 & 1.32 & 0.02 & Wierzbowski et al., 2012 \\
\hline & 237 & 46.75 & 1.18 & 0.01 & Wierzbowski et al., 2012 \\
\hline & 297 & 46.25 & 1.56 & 0.02 & Wierzbowski et al., 2012 \\
\hline & 298 & 46.25 & 1.48 & 0.01 & Wierzbowski et al., 2012 \\
\hline & 305 & 46.25 & 1.18 & 0.01 & Wierzbowski et al., 2012 \\
\hline & 307 & 46.25 & 1.31 & 0.02 & Wierzbowski et al., 2012 \\
\hline & 308 & 46.25 & 1.26 & 0.02 & Wierzbowski et al., 2012 \\
\hline & 260 & 45.75 & 1.34 & d.1. & Wierzbowski et al., 2012 \\
\hline & 261 & 45.75 & 1.36 & 0.01 & Wierzbowski et al., 2012 \\
\hline & 262 & 45.75 & 1.13 & 0.02 & Wierzbowski et al., 2012 \\
\hline & 264 & 45.75 & 1.25 & 0.01 & Wierzbowski et al., 2012 \\
\hline & 252 & 44.75 & 1.22 & 0.02 & Wierzbowski et al., 2012 \\
\hline & 253 & 44.75 & 1.27 & 0.02 & Wierzbowski et al., 2012 \\
\hline & 254 & 44.75 & 1.27 & d.1. & Wierzbowski et al., 2012 \\
\hline & 279 & 44.75 & 1.37 & 0.01 & Wierzbowski et al., 2012 \\
\hline & 256 & 44.25 & 1.35 & 0.04 & Wierzbowski et al., 2012 \\
\hline & 257 & 44.25 & 1.34 & 0.01 & Wierzbowski et al., 2012 \\
\hline & 272 & 44.25 & 1.26 & 0.04 & Wierzbowski et al., 2012 \\
\hline & 273 & 44.25 & 1.16 & 0.02 & Wierzbowski et al., 2012 \\
\hline & 238 & 42.17 & 1.30 & 0.08 & Wierzbowski et al., 2012 \\
\hline & 249 & 42.17 & 1.57 & 0.04 & Wierzbowski et al., 2012 \\
\hline & 251 & 42.17 & 1.31 & 0.07 & Wierzbowski et al., 2012 \\
\hline & 282 & 42.17 & 1.36 & 0.02 & Wierzbowski et al., 2012 \\
\hline & 283 & 42.17 & 1.32 & d.1. & Wierzbowski et al., 2012 \\
\hline & 243 & 41.50 & 1.28 & 0.02 & Wierzbowski et al., 2012 \\
\hline & 245 & 41.50 & 1.30 & 0.02 & Wierzbowski et al., 2012 \\
\hline & 246 & 41.50 & 1.32 & 0.03 & Wierzbowski et al., 2012 \\
\hline & 287 & 41.50 & 1.26 & 0.04 & Wierzbowski et al., 2012 \\
\hline & BB45 & 38.89 & 1.51 & d.1. & Wierzbowski et al., 2012 \\
\hline & BB43 & 38.89 & 1.48 & d.l. & Wierzbowski et al., 2012 \\
\hline & BB42 & 38.89 & 1.57 & d.1. & Wierzbowski et al., 2012 \\
\hline & BB59 & 38.89 & 1.40 & d.1. & Wierzbowski et al., 2012 \\
\hline
\end{tabular}




\begin{tabular}{|c|c|c|c|c|c|}
\hline & BB4 & 38.89 & 1.53 & d.l. & Wierzbowski et al., 2012 \\
\hline & BB31 & 38.41 & 1.47 & d.l. & Wierzbowski et al., 2012 \\
\hline & BB17 & 38.29 & 1.53 & d.l. & Wierzbowski et al., 2012 \\
\hline & BB19 & 38.20 & 1.51 & d.l. & Wierzbowski et al., 2012 \\
\hline & BB54 & 37.79 & 1.60 & d.l. & Wierzbowski et al., 2012 \\
\hline & BB8 & 37.79 & 1.58 & d.l. & Wierzbowski et al., 2012 \\
\hline & BB46 & 37.71 & 1.50 & d.l. & Wierzbowski et al., 2012 \\
\hline & BB32 & 37.71 & 1.56 & d.l. & Wierzbowski et al., 2012 \\
\hline & BB29 & 35.63 & 1.87 & d.l. & Wierzbowski et al., 2012 \\
\hline & BB15 & 35.39 & 1.48 & d.l. & Wierzbowski et al., 2012 \\
\hline & BB52 & 35.32 & 1.90 & d.l. & Wierzbowski et al., 2012 \\
\hline & BB13 & 35.32 & 1.67 & d.l. & Wierzbowski et al., 2012 \\
\hline & BB38 & 34.29 & 1.48 & d.l. & Wierzbowski et al., 2012 \\
\hline & BB35 & 34.29 & 1.79 & d.l. & Wierzbowski et al., 2012 \\
\hline & BB6 & 34.29 & 1.53 & d.l. & Wierzbowski et al., 2012 \\
\hline & $\mathrm{BB} 10$ & 34.27 & 1.39 & d.l. & Wierzbowski et al., 2012 \\
\hline & BB125 & 33.80 & 1.46 & 0.02 & Wierzbowski et al., 2012 \\
\hline & BB82 & 33.80 & 1.59 & d.l. & Wierzbowski et al., 2012 \\
\hline & BB40 & 33.80 & 1.58 & d.l. & Wierzbowski et al., 2012 \\
\hline & BB5 & 33.33 & 1.49 & d.l. & Wierzbowski et al., 2012 \\
\hline & BB127 & 33.21 & 1.33 & 0.04 & Wierzbowski et al., 2012 \\
\hline & BB36 & 33.16 & 1.35 & d.l. & Wierzbowski et al., 2012 \\
\hline & BB44 & 33.12 & 1.46 & 0.01 & Wierzbowski et al., 2012 \\
\hline & $\mathrm{BB} 25$ & 32.65 & 1.61 & 0.05 & Wierzbowski et al., 2012 \\
\hline & BB47 & 32.65 & 1.43 & 0.01 & Wierzbowski et al., 2012 \\
\hline & BB60 & 32.65 & 1.44 & 0.01 & Wierzbowski et al., 2012 \\
\hline & BB48 & 32.38 & 1.55 & d.l. & Wierzbowski et al., 2012 \\
\hline & $\mathrm{BB} 7$ & 32.36 & 1.63 & d.1. & Wierzbowski et al., 2012 \\
\hline & BB58 & 32.35 & 1.53 & 0.02 & Wierzbowski et al., 2012 \\
\hline Bearreraig & Bea_54 & 27.43 & 1.75 & 0.01 & present study \\
\hline Bearreraig & Bea_54 & 27.43 & 1.71 & 0.01 & present study \\
\hline Bearreraig & Bea_50 & 27.37 & 1.31 & 0.01 & present study \\
\hline Bearreraig & Bea_50 & 27.37 & 1.40 & 0.01 & present study \\
\hline Bearreraig & Bea_44 & 27.29 & 1.55 & 0.01 & present study \\
\hline Bearreraig & Bea_44 & 27.29 & 1.74 & 0.01 & present study \\
\hline Bearreraig & Bea_36 & 27.24 & 1.70 & 0.02 & present study \\
\hline Bearreraig & Bea_36 & 27.24 & 1.77 & 0.01 & present study \\
\hline Bearreraig & Bea_38 & 27.22 & 1.97 & 0.03 & present study \\
\hline Bearreraig & Bea_38 & 27.22 & 1.73 & 0.02 & present study \\
\hline Bearreraig & Bea_35 & 27.17 & 1.64 & 0.00 & present study \\
\hline Bearreraig & Bea_35 & 27.17 & 1.68 & 0.00 & present study \\
\hline Bearreraig & Bea_32 & 26.69 & 1.56 & 0.02 & present study \\
\hline Bearreraig & Bea_32 & 26.69 & 1.39 & 0.01 & present study \\
\hline Bearreraig & Bea_28 & 26.58 & 1.39 & 0.00 & present study \\
\hline Bearreraig & Bea_28 & 26.58 & 1.53 & 0.01 & present study \\
\hline Bearreraig & Bea_30 & 26.56 & 1.39 & 0.01 & present study \\
\hline Bearreraig & Bea_30 & 26.56 & 1.56 & 0.01 & present study \\
\hline Bearreraig & Bea_22 & 26.40 & 1.50 & 0.01 & present study \\
\hline Bearreraig & Bea_21 & 26.40 & 1.47 & 0.01 & present study \\
\hline
\end{tabular}




\begin{tabular}{|c|c|c|c|c|c|}
\hline Bearreraig & Bea_21 & 26.40 & 1.45 & 0.01 & present study \\
\hline Bearreraig & Bea_22 & 26.40 & 1.71 & 0.01 & present study \\
\hline Bearreraig & Bea_23 & 26.39 & 1.33 & 0.01 & present study \\
\hline Bearreraig & Bea_23 & 26.39 & 1.35 & 0.01 & present study \\
\hline Bearreraig & Bea_19 & 26.13 & 1.41 & 0.01 & present study \\
\hline Bearreraig & Bea_19 & 26.13 & 1.33 & 0.01 & present study \\
\hline Bearreraig & Bea_17 & 26.11 & 1.26 & 0.01 & present study \\
\hline Bearreraig & Bea_17 & 26.11 & 1.33 & 0.01 & present study \\
\hline Bearreraig & Bea_18 & 26.11 & 1.37 & 0.00 & present study \\
\hline Bearreraig & Bea_18 & 26.11 & 1.48 & 0.00 & present study \\
\hline Bearreraig & Bea_15 & 25.53 & 1.47 & 0.00 & present study \\
\hline Bearreraig & Bea_15 & 25.53 & 1.51 & 0.01 & present study \\
\hline Bearreraig & Bea_13 & 25.46 & 1.43 & 0.01 & present study \\
\hline Bearreraig & Bea_13 & 25.46 & 1.65 & 0.01 & present study \\
\hline Bearreraig & Bea_12 & 25.39 & 1.46 & 0.01 & present study \\
\hline Bearreraig & Bea_12 & 25.39 & 1.47 & 0.01 & present study \\
\hline Bearreraig & Bea_10 & 25.36 & 1.90 & 0.02 & present study \\
\hline Bearreraig & Bea_10 & 25.36 & 1.88 & 0.01 & present study \\
\hline Bearreraig & Bea_4 & 25.35 & 1.37 & 0.01 & present study \\
\hline Bearreraig & Bea_4 & 25.35 & 1.38 & 0.01 & present study \\
\hline Bearreraig & Bea_3 & 25.35 & 1.51 & 0.01 & present study \\
\hline Bearreraig & Bea_3 & 25.35 & 1.49 & 0.01 & present study \\
\hline Bearreraig & Bea 2 & 25.32 & 1.50 & 0.01 & present study \\
\hline Bearreraig & Bea_2 & 25.32 & 1.57 & 0.01 & present study \\
\hline Bearreraig & Bea_1 & 25.31 & 1.48 & 0.01 & present study \\
\hline Bearreraig & Bea_1 & 25.31 & 1.95 & 0.10 & present study \\
\hline Bearreraig & Bea_6 & 25.30 & 1.78 & 0.01 & present study \\
\hline Bearreraig & Bea_6 & 25.30 & 2.11 & 0.03 & present study \\
\hline Bearreraig & Bea_7 & 25.29 & 1.92 & 0.05 & present study \\
\hline Bearreraig & Bea_7 & 25.29 & 1.61 & 0.02 & present study \\
\hline Bearreraig & Bea_144 & 23.50 & 1.72 & 0.01 & present study \\
\hline Bearreraig & Bea_144 & 23.50 & 1.82 & 0.01 & present study \\
\hline Bearreraig & Bea 141 & 23.33 & 1.68 & 0.00 & present study \\
\hline Bearreraig & Bea_141 & 23.33 & 1.61 & 0.01 & present study \\
\hline Bearreraig & Bea_142 & 23.33 & 2.04 & 0.01 & present study \\
\hline Bearreraig & Bea_142 & 23.33 & 1.81 & 0.01 & present study \\
\hline Bearreraig & Bea_139 & 23.20 & 1.99 & 0.01 & present study \\
\hline Bearreraig & Bea_139 & 23.20 & 1.91 & 0.01 & present study \\
\hline Bearreraig & Bea_134 & 23.06 & 1.72 & 0.02 & present study \\
\hline Bearreraig & Bea_134 & 23.06 & 1.91 & 0.02 & present study \\
\hline Bearreraig & Bea_132 & 22.97 & 1.31 & 0.01 & present study \\
\hline Bearreraig & Bea_132 & 22.97 & 1.26 & 0.01 & present study \\
\hline Bearreraig & Bea 131 & 22.76 & 1.62 & 0.01 & present study \\
\hline Bearreraig & Bea_131 & 22.76 & 1.68 & 0.01 & present study \\
\hline Bearreraig & Bea_136 & 22.74 & 1.64 & 0.01 & present study \\
\hline Bearreraig & Bea_136 & 22.74 & 1.73 & 0.01 & present study \\
\hline Bearreraig & Bea_130 & 22.71 & 1.62 & 0.01 & present study \\
\hline Bearreraig & Bea_130 & 22.71 & 1.64 & 0.01 & present study \\
\hline Bearreraig & Bea_128 & 22.69 & 1.54 & 0.01 & present study \\
\hline
\end{tabular}




\begin{tabular}{|c|c|c|c|c|c|}
\hline Bearreraig & Bea_128 & 22.69 & 1.49 & 0.01 & present study \\
\hline Bearreraig & Bea_118 & 21.72 & 1.52 & 0.02 & present study \\
\hline Bearreraig & Bea_118 & 21.72 & 1.64 & 0.01 & present study \\
\hline Bearreraig & Bea_116 & 21.63 & 1.92 & 0.01 & present study \\
\hline Bearreraig & Bea_116 & 21.63 & 1.89 & 0.02 & present study \\
\hline Bearreraig & Bea_117 & 21.54 & 1.63 & 0.01 & present study \\
\hline Bearreraig & Bea_117 & 21.54 & 1.79 & 0.01 & present study \\
\hline Bearreraig & Bea_122 & 21.40 & 1.62 & 0.02 & present study \\
\hline Bearreraig & Bea_122 & 21.40 & 1.64 & 0.02 & present study \\
\hline Bearreraig & Bea_121 & 21.40 & 1.50 & 0.02 & present study \\
\hline Bearreraig & Bea_121 & 21.40 & 1.61 & 0.01 & present study \\
\hline Bearreraig & Bea_120 & 21.39 & 1.69 & 0.01 & present study \\
\hline Bearreraig & Bea_120 & 21.39 & 1.81 & 0.01 & present study \\
\hline Bearreraig & Bea_124 & 21.13 & 1.60 & 0.01 & present study \\
\hline Bearreraig & Bea_124 & 21.13 & 1.76 & 0.01 & present study \\
\hline Bearreraig & Bea_126 & 21.08 & 1.58 & 0.02 & present study \\
\hline Bearreraig & Bea_126 & 21.08 & 1.62 & 0.01 & present study \\
\hline Bearreraig & Bea_125 & 21.06 & 2.01 & 0.01 & present study \\
\hline Bearreraig & Bea_125 & 21.06 & 1.86 & 0.01 & present study \\
\hline Bearreraig & Bea_74 & 20.53 & 1.63 & 0.01 & present study \\
\hline Bearreraig & Bea_74 & 20.53 & 1.64 & 0.02 & present study \\
\hline Bearreraig & Bea_73 & 20.53 & 1.85 & 0.01 & present study \\
\hline Bearreraig & Bea_73 & 20.53 & 1.79 & 0.01 & present study \\
\hline Bearreraig & Bea_81 & 20.53 & 1.80 & 0.03 & present study \\
\hline Bearreraig & Bea_72 & 20.53 & 1.85 & 0.01 & present study \\
\hline Bearreraig & Bea_81 & 20.53 & 1.87 & 0.02 & present study \\
\hline Bearreraig & Bea_72 & 20.53 & 1.93 & 0.01 & present study \\
\hline Bearreraig & Bea_80 & 20.34 & 1.51 & 0.01 & present study \\
\hline Bearreraig & Bea_80 & 20.34 & 1.79 & 0.01 & present study \\
\hline Bearreraig & Bea_78 & 20.19 & 2.00 & 0.02 & present study \\
\hline Bearreraig & Bea_78 & 20.19 & 1.97 & 0.01 & present study \\
\hline Bearreraig & Bea_77 & 20.03 & 1.71 & 0.01 & present study \\
\hline Bearreraig & Bea_77 & 20.03 & 1.72 & 0.01 & present study \\
\hline Bearreraig & Bea_82 & 19.98 & 1.70 & 0.03 & present study \\
\hline Bearreraig & Bea_82 & 19.98 & 1.92 & 0.04 & present study \\
\hline Bearreraig & Bea_88 & 19.98 & 1.74 & 0.02 & present study \\
\hline Bearreraig & Bea_88 & 19.98 & 1.54 & 0.02 & present study \\
\hline Bearreraig & Bea_89 & 19.98 & 2.16 & 0.01 & present study \\
\hline Bearreraig & Bea_89 & 19.98 & 2.08 & 0.01 & present study \\
\hline Bearreraig & Bea_84 & 19.97 & 1.54 & 0.01 & present study \\
\hline Bearreraig & Bea_84 & 19.97 & 1.53 & 0.01 & present study \\
\hline Bearreraig & Bea_91 & 19.96 & 1.60 & 0.02 & present study \\
\hline Bearreraig & Bea_91 & 19.96 & 1.70 & 0.02 & present study \\
\hline Bearreraig & Bea_90 & 19.95 & 1.91 & 0.01 & present study \\
\hline Bearreraig & Bea_90 & 19.95 & 1.86 & 0.01 & present study \\
\hline Bearreraig & Bea_85 & 19.94 & 1.40 & 0.01 & present study \\
\hline Bearreraig & Bea_85 & 19.94 & 1.42 & 0.01 & present study \\
\hline Bearreraig & Bea_111 & 19.94 & 1.91 & 0.01 & present study \\
\hline Bearreraig & Bea_111 & 19.94 & 1.95 & 0.01 & present study \\
\hline
\end{tabular}




\begin{tabular}{|c|c|c|c|c|c|}
\hline Bearreraig & Bea_113 & 19.92 & 1.69 & 0.01 & present study \\
\hline Bearreraig & Bea_114 & 19.92 & 1.88 & 0.01 & present study \\
\hline Bearreraig & Bea_114 & 19.92 & 1.86 & 0.01 & present study \\
\hline Bearreraig & Bea_113 & 19.92 & 1.76 & 0.01 & present study \\
\hline Bearreraig & Bea_110 & 19.91 & 1.65 & 0.01 & present study \\
\hline Bearreraig & Bea_110 & 19.91 & 1.79 & 0.01 & present study \\
\hline Bearreraig & Bea_109 & 19.90 & 1.56 & 0.01 & present study \\
\hline Bearreraig & Bea_109 & 19.90 & 1.68 & 0.02 & present study \\
\hline Bearreraig & Bea_102 & 19.89 & 1.64 & 0.01 & present study \\
\hline Bearreraig & Bea_102 & 19.89 & 1.64 & 0.01 & present study \\
\hline Bearreraig & Bea_100 & 19.89 & 1.83 & 0.01 & present study \\
\hline Bearreraig & Bea_100 & 19.89 & 1.80 & 0.01 & present study \\
\hline Bearreraig & Bea_105 & 19.88 & 1.78 & 0.01 & present study \\
\hline Bearreraig & Bea_105 & 19.88 & 1.77 & 0.01 & present study \\
\hline Bearreraig & Bea_104 & 19.88 & 2.04 & 0.01 & present study \\
\hline Bearreraig & Bea_104 & 19.88 & 1.98 & 0.01 & present study \\
\hline Bearreraig & Bea_97 & 19.83 & 1.75 & 0.02 & present study \\
\hline Bearreraig & Bea_97 & 19.83 & 1.68 & 0.01 & present study \\
\hline Bearreraig & Bea_93 & 19.79 & 1.72 & 0.01 & present study \\
\hline Bearreraig & Bea_93 & 19.79 & 1.78 & 0.01 & present study \\
\hline Bearreraig & Bea_94 & 19.76 & 1.83 & 0.01 & present study \\
\hline Bearreraig & Bea_94 & 19.76 & 1.73 & 0.02 & present study \\
\hline Bearreraig & Bea_92 & 19.75 & 1.49 & 0.01 & present study \\
\hline Bearreraig & Bea_92 & 19.75 & 1.72 & 0.01 & present study \\
\hline Peak & P3 & 17.94 & 1.55 & 0.01 & McArthur et al., 2000 \\
\hline Ravenscar & Rave_115 & 17.90 & 2.17 & 0.02 & present study \\
\hline Ravenscar & Rave_115 & 17.90 & 2.44 & 0.10 & present study \\
\hline Peak & P4 & 17.88 & 1.68 & 0.01 & McArthur et al., 2000 \\
\hline Peak & P5 & 17.79 & 1.67 & 0.01 & McArthur et al., 2000 \\
\hline Ravenscar & Rave_112 & 17.78 & 2.02 & 0.02 & present study \\
\hline Ravenscar & Rave_112 & 17.78 & 1.65 & 0.02 & present study \\
\hline Peak & P7 & 17.75 & 1.93 & 0.16 & McArthur et al., 2000 \\
\hline Ravenscar & Rave_109 & 17.74 & 2.10 & 0.01 & present study \\
\hline Ravenscar & Rave_109 & 17.74 & 2.06 & 0.01 & present study \\
\hline Ravenscar & Rave_111 & 17.73 & 1.82 & 0.03 & present study \\
\hline Ravenscar & Rave_111 & 17.73 & 2.07 & 0.03 & present study \\
\hline Peak & P8 & 17.72 & 1.61 & 0.02 & McArthur et al., 2000 \\
\hline Ravenscar & Rave_108 & 17.70 & 2.40 & 0.02 & present study \\
\hline Ravenscar & Rave_108 & 17.70 & 2.36 & 0.04 & present study \\
\hline Ravenscar & Rave_105 & 17.68 & 1.73 & 0.01 & present study \\
\hline Ravenscar & Rave_105 & 17.68 & 1.99 & 0.02 & present study \\
\hline Ravenscar & Rave_106 & 17.68 & 2.12 & 0.04 & present study \\
\hline Ravenscar & Rave_104 & 17.64 & 2.20 & 0.01 & present study \\
\hline Ravenscar & Rave_104 & 17.64 & 2.05 & 0.01 & present study \\
\hline Ravenscar & Rave_103 & 17.64 & 1.86 & 0.01 & present study \\
\hline Ravenscar & Rave_103 & 17.64 & 1.66 & 0.01 & present study \\
\hline Ravenscar & Rave_100 & 17.56 & 1.78 & 0.01 & present study \\
\hline Ravenscar & Rave_100 & 17.56 & 1.65 & 0.01 & present study \\
\hline Ravenscar & Rave_101 & 17.55 & 2.06 & 0.01 & present study \\
\hline
\end{tabular}




\begin{tabular}{|c|c|c|c|c|c|}
\hline Ravenscar & Rave_101 & 17.55 & 1.79 & 0.02 & present study \\
\hline Ravenscar & Rave_114 & 17.51 & 1.82 & 0.01 & present study \\
\hline Ravenscar & Rave_114 & 17.51 & 1.74 & 0.02 & present study \\
\hline Peak & $\mathrm{P} 10$ & 17.51 & 1.67 & 0.01 & McArthur et al., 2000 \\
\hline Ravenscar & Rave_99 & 17.49 & 1.72 & 0.01 & present study \\
\hline Ravenscar & Rave_99 & 17.49 & 1.85 & 0.01 & present study \\
\hline Peak & P11 & 17.46 & 1.85 & 0.02 & McArthur et al., 2000 \\
\hline Peak & P12 & 17.40 & 1.61 & 0.02 & McArthur et al., 2000 \\
\hline Peak & P14 & 17.39 & 1.50 & 0.01 & McArthur et al., 2000 \\
\hline Peak & P15 & 17.39 & 1.83 & 0.03 & McArthur et al., 2000 \\
\hline Ravenscar & Rave_95 & 17.34 & 1.77 & 0.01 & present study \\
\hline Ravenscar & Rave_95 & 17.34 & 1.94 & 0.01 & present study \\
\hline Ravenscar & Rave_93 & 17.31 & 2.24 & 0.01 & present study \\
\hline Ravenscar & Rave_93 & 17.31 & 1.86 & 0.02 & present study \\
\hline Ravenscar & Rave_94 & 17.31 & 2.20 & 0.01 & present study \\
\hline Ravenscar & Rave_94 & 17.31 & 1.98 & 0.01 & present study \\
\hline Ravenscar & Rave_89 & 17.27 & 2.28 & 0.01 & present study \\
\hline Ravenscar & Rave_77 & 17.26 & 1.98 & 0.01 & present study \\
\hline Ravenscar & Rave_77 & 17.26 & 1.81 & 0.01 & present study \\
\hline Ravenscar & Rave_76 & 17.26 & 1.78 & 0.01 & present study \\
\hline Ravenscar & Rave_76 & 17.26 & 1.46 & 0.07 & present study \\
\hline Ravenscar & Rave_86 & 17.20 & 2.12 & 0.01 & present study \\
\hline Ravenscar & Rave_86 & 17.20 & 1.95 & 0.02 & present study \\
\hline Peak & P17 & 17.19 & 1.38 & 0.01 & McArthur et al., 2000 \\
\hline Ravenscar & Rave_78 & 17.18 & 2.05 & 0.02 & present study \\
\hline Ravenscar & Rave_78 & 17.18 & 1.87 & 0.02 & present study \\
\hline Ravenscar & Rave_79 & 17.18 & 2.35 & 0.01 & present study \\
\hline Ravenscar & Rave_79 & 17.18 & 1.95 & 0.01 & present study \\
\hline Ravenscar & Rave_74 & 17.16 & 2.15 & 0.01 & present study \\
\hline Ravenscar & Rave_74 & 17.16 & 2.14 & 0.02 & present study \\
\hline Ravenscar & Rave_80 & 17.15 & 1.94 & 0.01 & present study \\
\hline Ravenscar & Rave_80 & 17.15 & 2.05 & 0.01 & present study \\
\hline Ravenscar & Rave_81 & 17.13 & 2.15 & 0.01 & present study \\
\hline Ravenscar & Rave_81 & 17.13 & 2.04 & 0.01 & present study \\
\hline Peak & $\mathrm{P} 20$ & 17.13 & 1.64 & 0.01 & McArthur et al., 2000 \\
\hline Ravenscar & Rave_84 & 17.10 & 2.13 & 0.01 & present study \\
\hline Ravenscar & Rave_84 & 17.10 & 2.05 & 0.02 & present study \\
\hline Peak & $\mathrm{P} 23$ & 16.92 & 1.63 & 0.00 & McArthur et al., 2000 \\
\hline Peak & $\mathrm{P} 24$ & 16.92 & 1.83 & 0.01 & McArthur et al., 2000 \\
\hline Ravenscar & Rave_71 & 16.89 & 1.71 & 0.01 & present study \\
\hline Ravenscar & Rave_71 & 16.89 & 1.65 & 0.03 & present study \\
\hline Ravenscar & Rave_72 & 16.85 & 1.85 & 0.01 & present study \\
\hline Ravenscar & Rave_72 & 16.85 & 1.96 & 0.01 & present study \\
\hline Ravenscar & Rave_69 & 16.84 & 1.73 & 0.02 & present study \\
\hline Ravenscar & Rave_69 & 16.84 & 1.83 & 0.03 & present study \\
\hline Ravenscar & Rave_73 & 16.83 & 2.03 & 0.06 & present study \\
\hline Ravenscar & Rave_73 & 16.83 & 1.73 & 0.10 & present study \\
\hline Ravenscar & Rave_68 & 16.81 & 2.27 & 0.01 & present study \\
\hline Ravenscar & Rave_68 & 16.81 & 2.09 & 0.01 & present study \\
\hline
\end{tabular}




\begin{tabular}{|c|c|c|c|c|c|}
\hline Ravenscar & Rave_66 & 16.79 & 1.73 & 0.01 & present study \\
\hline Ravenscar & Rave_66 & 16.79 & 2.00 & 0.02 & present study \\
\hline Ravenscar & Rave_65 & 16.75 & 2.00 & 0.01 & present study \\
\hline Ravenscar & Rave_65 & 16.75 & 2.19 & 0.01 & present study \\
\hline Saltwick Bay & S424 & 16.75 & 1.43 & 0.02 & McArthur et al., 2000 \\
\hline Ravenscar & Rave_64 & 16.73 & 1.97 & 0.01 & present study \\
\hline Ravenscar & Rave_64 & 16.73 & 1.84 & 0.05 & present study \\
\hline Saltwick Bay & S437A & 16.71 & 1.32 & 0.03 & McArthur et al., 2000 \\
\hline Saltwick Bay & S437B & 16.71 & 1.61 & 0.00 & McArthur et al., 2000 \\
\hline Ravenscar & Rave_62 & 16.69 & 2.13 & 0.01 & present study \\
\hline Ravenscar & Rave_62 & 16.69 & 2.13 & 0.01 & present study \\
\hline Ravenscar & Rave_63 & 16.69 & 2.05 & 0.02 & present study \\
\hline Ravenscar & Rave_63 & 16.69 & 2.10 & 0.02 & present study \\
\hline Saltwick Bay & S436 & 16.67 & 1.87 & 0.01 & McArthur et al., 2000 \\
\hline Peak & $\mathrm{P} 25$ & 16.64 & 1.73 & 0.03 & McArthur et al., 2000 \\
\hline Ravenscar & Rave_61 & 16.64 & 1.97 & 0.01 & present study \\
\hline Ravenscar & Rave_61 & 16.64 & 1.89 & 0.01 & present study \\
\hline Ravenscar & Rave_60 & 16.60 & 1.63 & 0.00 & present study \\
\hline Ravenscar & Rave_60 & 16.60 & 1.64 & 0.01 & present study \\
\hline Ravenscar & Rave_58 & 16.55 & 2.03 & 0.01 & present study \\
\hline Ravenscar & Rave_58 & 16.55 & 1.97 & 0.01 & present study \\
\hline Peak & $\mathrm{P} 28$ & 16.55 & 1.84 & 0.01 & McArthur et al., 2000 \\
\hline Ravenscar & Rave_59 & 16.53 & 2.03 & 0.00 & present study \\
\hline Ravenscar & Rave_59 & 16.53 & 1.86 & 0.01 & present study \\
\hline Ravenscar & Rave_57 & 16.53 & 1.96 & 0.01 & present study \\
\hline Ravenscar & Rave_57 & 16.53 & 1.96 & 0.01 & present study \\
\hline Peak & P29 & 16.52 & 1.52 & 0.01 & McArthur et al., 2000 \\
\hline Ravenscar & Rave_56 & 16.49 & 1.96 & 0.01 & present study \\
\hline Ravenscar & Rave_56 & 16.49 & 1.91 & 0.01 & present study \\
\hline Saltwick Bay & $\mathrm{S} 435 \mathrm{~A}$ & 16.48 & 1.53 & 0.01 & McArthur et al., 2000 \\
\hline Saltwick Bay & $\mathrm{S} 432 \mathrm{~A}$ & 16.45 & 1.59 & 0.00 & McArthur et al., 2000 \\
\hline Ravenscar & Rave_54 & 16.45 & 2.10 & 0.01 & present study \\
\hline Ravenscar & Rave_54 & 16.45 & 2.39 & 0.01 & present study \\
\hline Ravenscar & Rave_52 & 16.44 & 2.51 & 0.01 & present study \\
\hline Ravenscar & Rave_52 & 16.44 & 2.33 & 0.01 & present study \\
\hline Ravenscar & Rave_48 & 16.43 & 1.40 & 0.05 & present study \\
\hline Saltwick Bay & S428 & 16.41 & 1.49 & 0.01 & McArthur et al., 2000 \\
\hline Ravenscar & Rave_49 & 16.40 & 1.81 & 0.02 & present study \\
\hline Ravenscar & Rave_49 & 16.40 & 1.35 & 0.02 & present study \\
\hline Ravenscar & Rave_51 & 16.39 & 2.32 & 0.01 & present study \\
\hline Ravenscar & Rave_51 & 16.39 & 1.90 & 0.01 & present study \\
\hline Saltwick Bay & $\mathrm{S} 423 \mathrm{~A}$ & 16.36 & 1.62 & 0.01 & McArthur et al., 2000 \\
\hline Ravenscar & Rave_44 & 16.34 & 1.90 & 0.01 & present study \\
\hline Ravenscar & Rave_44 & 16.34 & 1.96 & 0.01 & present study \\
\hline Saltwick Bay & $\mathrm{S} 422 \mathrm{~B}$ & 16.33 & 1.49 & 0.01 & McArthur et al., 2000 \\
\hline Saltwick Bay & S421A & 16.32 & 1.63 & 0.00 & McArthur et al., 2000 \\
\hline Ravenscar & Rave_47 & 16.32 & 2.24 & 0.01 & present study \\
\hline Ravenscar & Rave_47 & 16.32 & 2.06 & 0.02 & present study \\
\hline Ravenscar & Rave_43 & 16.31 & 1.75 & 0.02 & present study \\
\hline
\end{tabular}




\begin{tabular}{|c|c|c|c|c|c|}
\hline Ravenscar & Rave_43 & 16.31 & 1.79 & 0.02 & present study \\
\hline Ravenscar & Rave_42 & 16.31 & 1.76 & 0.01 & present study \\
\hline Ravenscar & Rave_42 & 16.31 & 1.87 & 0.02 & present study \\
\hline Ravenscar & Rave_38 & 16.30 & 1.92 & 0.01 & present study \\
\hline Ravenscar & Rave_38 & 16.30 & 2.13 & 0.01 & present study \\
\hline Ravenscar & Rave_40 & 16.30 & 2.23 & 0.01 & present study \\
\hline Ravenscar & Rave_40 & 16.30 & 1.92 & 0.02 & present study \\
\hline Ravenscar & Rave_41 & 16.30 & 1.78 & 0.01 & present study \\
\hline Ravenscar & Rave_41 & 16.30 & 1.73 & 0.01 & present study \\
\hline Ravenscar & Rave_37 & 16.29 & 1.97 & 0.01 & present study \\
\hline Ravenscar & Rave_37 & 16.29 & 2.10 & 0.01 & present study \\
\hline Ravenscar & Rave_35 & 16.29 & 1.94 & 0.01 & present study \\
\hline Ravenscar & Rave_35 & 16.29 & 1.98 & 0.01 & present study \\
\hline Ravenscar & Rave_36 & 16.29 & 1.73 & 0.01 & present study \\
\hline Ravenscar & Rave_36 & 16.29 & 1.81 & 0.01 & present study \\
\hline Saltwick Bay & $\mathrm{S} 418 \mathrm{C}$ & 16.28 & 1.90 & 0.00 & McArthur et al., 2000 \\
\hline Ravenscar & Rave_32 & 16.26 & 2.04 & 0.01 & present study \\
\hline Ravenscar & Rave_32 & 16.26 & 1.84 & 0.01 & present study \\
\hline Ravenscar & Rave_29 & 16.25 & 2.01 & 0.01 & present study \\
\hline Ravenscar & Rave_29 & 16.25 & 2.09 & 0.02 & present study \\
\hline Ravenscar & Rave_34 & 16.25 & 1.99 & 0.01 & present study \\
\hline Ravenscar & Rave_34 & 16.25 & 2.28 & 0.01 & present study \\
\hline Saltwick Bay & S414B & 16.25 & 1.62 & 0.01 & McArthur et al., 2000 \\
\hline Ravenscar & Rave_25 & 16.24 & 2.24 & 0.01 & present study \\
\hline Ravenscar & Rave_25 & 16.24 & 2.09 & 0.01 & present study \\
\hline Ravenscar & Rave_23 & 16.23 & 2.07 & 0.01 & present study \\
\hline Ravenscar & Rave_23 & 16.23 & 2.13 & 0.01 & present study \\
\hline Ravenscar & Rave_28 & 16.22 & 2.06 & 0.01 & present study \\
\hline Ravenscar & Rave_28 & 16.22 & 2.09 & 0.02 & present study \\
\hline Ravenscar & Rave_19 & 16.22 & 2.12 & 0.01 & present study \\
\hline Ravenscar & Rave_19 & 16.22 & 2.11 & 0.01 & present study \\
\hline Saltwick Bay & S413A & 16.21 & 1.51 & 0.00 & McArthur et al., 2000 \\
\hline Ravenscar & Rave_18 & 16.21 & 1.91 & 0.01 & present study \\
\hline Ravenscar & Rave_18 & 16.21 & 1.77 & 0.02 & present study \\
\hline Ravenscar & Rave_16 & 16.18 & 1.72 & 0.01 & present study \\
\hline Ravenscar & Rave_16 & 16.18 & 2.06 & 0.02 & present study \\
\hline Ravenscar & Rave_15 & 16.18 & 1.72 & 0.02 & present study \\
\hline Ravenscar & Rave_15 & 16.18 & 1.90 & 0.02 & present study \\
\hline Ravenscar & Rave_14 & 16.18 & 2.10 & 0.02 & present study \\
\hline Ravenscar & Rave_14 & 16.18 & 1.90 & 0.03 & present study \\
\hline Saltwick Bay & S406 & 16.17 & 1.62 & 0.01 & McArthur et al., 2000 \\
\hline Saltwick Bay & Y06 51/110 & 16.13 & 1.77 & 0.00 & Li et al., 2012 \\
\hline Saltwick Bay & Y06 51/78 & 16.13 & 1.75 & 0.00 & Li et al., 2012 \\
\hline Saltwick Bay & Y06A 51/75 & 16.13 & 1.85 & 0.00 & Li et al., 2012 \\
\hline Saltwick Bay & Y06B 51/75 & 16.13 & 1.86 & 0.00 & Li et al., 2012 \\
\hline Saltwick Bay & $\mathrm{S} 401$ & 16.13 & 1.62 & 0.00 & McArthur et al., 2000 \\
\hline Saltwick Bay & Y06 51/60 & 16.13 & 1.90 & 0.00 & Li et al., 2012 \\
\hline Saltwick Bay & Y06A 51 base & 16.11 & 1.79 & 0.00 & Li et al., 2012 \\
\hline Saltwick Bay & Y06B 51 base & 16.11 & 1.74 & 0.00 & Li et al., 2012 \\
\hline
\end{tabular}




\begin{tabular}{|c|c|c|c|c|c|}
\hline Saltwick Bay & Y06A 49/620 & 16.11 & 1.78 & 0.00 & Li et al., 2012 \\
\hline Saltwick Bay & Y06B 49/620 & 16.11 & 1.88 & 0.00 & Li et al., 2012 \\
\hline Saltwick Bay & Y06C 49/620 & 16.11 & 1.75 & 0.00 & Li et al., 2012 \\
\hline Saltwick Bay & S327 & 16.11 & 1.50 & 0.01 & McArthur et al., 2000 \\
\hline Saltwick Bay & Y06 49/592 & 16.11 & 2.05 & 0.00 & Li et al., 2012 \\
\hline Saltwick Bay & Y06 49/519 & 16.09 & 1.73 & 0.00 & Li et al., 2012 \\
\hline Saltwick Bay & Y06 49/475 & 16.09 & 1.99 & 0.00 & Li et al., 2012 \\
\hline Saltwick Bay & S340 & 16.08 & 1.60 & 0.01 & McArthur et al., 2000 \\
\hline Saltwick Bay & Y06 49/420 & 16.08 & 1.77 & 0.00 & Li et al., 2012 \\
\hline Ravenscar & Rave_13 & 16.07 & 1.59 & 0.01 & present study \\
\hline Ravenscar & Rave_13 & 16.07 & 1.80 & 0.02 & present study \\
\hline Ravenscar & Rave_12 & 16.06 & 2.17 & 0.01 & present study \\
\hline Ravenscar & Rave_12 & 16.06 & 2.13 & 0.01 & present study \\
\hline Ravenscar & Rave_11 & 16.06 & 1.91 & 0.01 & present study \\
\hline Ravenscar & Rave_11 & 16.06 & 1.74 & 0.04 & present study \\
\hline Ravenscar & Rave_10 & 16.06 & 1.61 & 0.01 & present study \\
\hline Ravenscar & Rave_10 & 16.06 & 1.72 & 0.02 & present study \\
\hline Saltwick Bay & S342 & 16.06 & 1.82 & 0.00 & McArthur et al., 2000 \\
\hline Saltwick Bay & S343 & 16.03 & 1.67 & 0.01 & McArthur et al., 2000 \\
\hline Ravenscar & Rave_9 & 16.02 & 2.01 & 0.01 & present study \\
\hline Ravenscar & Rave_9 & 16.02 & 1.98 & 0.01 & present study \\
\hline Ravenscar & Rave_8 & 16.01 & 2.10 & 0.01 & present study \\
\hline Ravenscar & Rave_8 & 16.01 & 2.18 & 0.02 & present study \\
\hline Saltwick Bay & Y06 49/63 & 16.01 & 1.74 & 0.00 & Li et al., 2012 \\
\hline Saltwick Bay & Y06 49/30 & 16.01 & 2.07 & 0.00 & Li et al., 2012 \\
\hline Ravenscar & Rave_7 & 16.01 & 1.91 & 0.01 & present study \\
\hline Ravenscar & Rave_7 & 16.01 & 1.80 & 0.01 & present study \\
\hline Saltwick Bay & Y06 49/25 & 16.00 & 1.71 & 0.00 & Li et al., 2012 \\
\hline Saltwick Bay & Y06 49/22 & 16.00 & 1.79 & 0.00 & Li et al., 2012 \\
\hline Saltwick Bay & S313A & 16.00 & 1.74 & 0.00 & McArthur et al., 2000 \\
\hline Ravenscar & Rave_6 & 15.99 & 1.75 & 0.01 & Li et al., 2012 \\
\hline Ravenscar & Rave_6 & 15.99 & 1.67 & 0.02 & Li et al., 2012 \\
\hline Saltwick Bay & Y06 47/529 & 15.99 & 1.62 & 0.00 & Li et al., 2012 \\
\hline Saltwick Bay & Y06 47/526 & 15.99 & 1.72 & 0.00 & Li et al., 2012 \\
\hline Saltwick Bay & Y06A 47/520 & 15.99 & 1.81 & 0.00 & Li et al., 2012 \\
\hline Saltwick Bay & Y06B 47/520 & 15.99 & 1.73 & 0.00 & Li et al., 2012 \\
\hline Saltwick Bay & Y06 47/492 & 15.98 & 1.91 & 0.00 & Li et al., 2012 \\
\hline Saltwick Bay & Y06 47/485 & 15.98 & 1.91 & 0.00 & Li et al., 2012 \\
\hline Ravenscar & Rave_4 & 15.97 & 1.80 & 0.01 & present study \\
\hline Ravenscar & Rave_4 & 15.97 & 1.62 & 0.02 & present study \\
\hline Ravenscar & Rave_1 & 15.97 & 1.90 & 0.00 & present study \\
\hline Ravenscar & Rave_1 & 15.97 & 1.79 & 0.01 & present study \\
\hline Ravenscar & Rave_2 & 15.97 & 2.05 & 0.01 & present study \\
\hline Ravenscar & Rave_2 & 15.97 & 1.93 & 0.02 & present study \\
\hline Saltwick Bay & S315 & 15.97 & 1.48 & 0.01 & McArthur et al., 2000 \\
\hline Saltwick Bay & Y06 47/416 & 15.96 & 1.94 & 0.00 & Li et al., 2012 \\
\hline Ravenscar & Rave_3 & 15.96 & 1.82 & 0.01 & present study \\
\hline Ravenscar & Rave_3 & 15.96 & 1.71 & 0.01 & present study \\
\hline Saltwick Bay & Y06 47/379 & 15.96 & 1.85 & 0.00 & Li et al., 2012 \\
\hline
\end{tabular}




\begin{tabular}{|c|c|c|c|c|c|}
\hline Saltwick Bay & Y06 47/307 & 15.94 & 1.62 & 0.00 & Li et al., 2012 \\
\hline Saltwick Bay & S319B & 15.93 & 1.74 & 0.01 & McArthur et al., 2000 \\
\hline Saltwick Bay & S310 & 15.88 & 1.65 & 0.00 & McArthur et al., 2000 \\
\hline Saltwick Bay & S321 & 15.85 & 1.65 & 0.01 & McArthur et al., 2000 \\
\hline Saltwick Bay & S306 & 15.82 & 1.65 & 0.00 & McArthur et al., 2000 \\
\hline Saltwick Bay & S302 & 15.80 & 1.43 & 0.01 & McArthur et al., 2000 \\
\hline Saltwick Bay & S14A & 15.80 & 1.99 & 0.02 & McArthur et al., 2000 \\
\hline Saltwick Bay & Y06 45/0 & 15.80 & 1.54 & 0.02 & Li et al., 2012 \\
\hline Saltwick Bay & S113 & 15.79 & 1.76 & 0.01 & McArthur et al., 2000 \\
\hline Saltwick Bay & Y06 43/718 & 15.78 & 1.55 & 0.00 & Li et al., 2012 \\
\hline Saltwick Bay & Y06 43/698 & 15.78 & 1.73 & 0.00 & Li et al., 2012 \\
\hline Saltwick Bay & Y06A 43/691 & 15.78 & 1.83 & 0.00 & Li et al., 2012 \\
\hline Saltwick Bay & Y06B 43/691 & 15.78 & 1.79 & 0.00 & Li et al., 2012 \\
\hline Saltwick Bay & Y06 43/671 & 15.77 & 1.55 & 0.00 & Li et al., 2012 \\
\hline Saltwick Bay & Y06 43/641 & 15.77 & 2.10 & 0.00 & Li et al., 2012 \\
\hline Saltwick Bay & Y06B 43/580 & 15.75 & 1.66 & 0.00 & Li et al., 2012 \\
\hline Saltwick Bay & S11A & 15.75 & 1.41 & 0.03 & McArthur et al., 2000 \\
\hline Saltwick Bay & Y06B 43/516 & 15.74 & 1.60 & 0.00 & Li et al., 2012 \\
\hline Saltwick Bay & Y06C 43/516 & 15.74 & 1.60 & 0.00 & Li et al., 2012 \\
\hline Saltwick Bay & Y06 43/515 & 15.74 & 1.67 & 0.00 & Li et al., 2012 \\
\hline Saltwick Bay & Y06A 43/510 & 15.74 & 1.56 & 0.00 & Li et al., 2012 \\
\hline Saltwick Bay & Y06B 43/510 & 15.74 & 1.87 & 0.00 & Li et al., 2012 \\
\hline Saltwick Bay & Y06 43/392 & 15.71 & 1.72 & 0.00 & Li et al., 2012 \\
\hline Saltwick Bay & S6B & 15.71 & 1.80 & 0.03 & McArthur et al., 2000 \\
\hline Saltwick Bay & Y06 43/387 & 15.71 & 1.78 & 0.00 & Li et al., 2012 \\
\hline Saltwick Bay & Y06 43/362 & 15.71 & 1.61 & 0.00 & Li et al., 2012 \\
\hline Saltwick Bay & Y06 43/326 & 15.70 & 1.65 & 0.01 & Li et al., 2012 \\
\hline Saltwick Bay & Y06A 43/310 & 15.70 & 1.60 & 0.00 & Li et al., 2012 \\
\hline Saltwick Bay & Y06B 43/310 & 15.70 & 1.70 & 0.00 & Li et al., 2012 \\
\hline Saltwick Bay & Y06D 43/310 & 15.70 & 1.72 & 0.00 & Li et al., 2012 \\
\hline Saltwick Bay & Y06A 43/307 & 15.69 & 1.77 & 0.00 & Li et al., 2012 \\
\hline Saltwick Bay & S9B & 15.69 & 1.51 & 0.02 & McArthur et al., 2000 \\
\hline Runswick & $\mathrm{R} 8$ & 15.68 & 1.78 & 0.03 & McArthur et al., 2000 \\
\hline Saltwick Bay & Y06 43/200 & 15.67 & 1.73 & 0.00 & Li et al., 2012 \\
\hline Runswick & R6A & 15.66 & 1.77 & 0.04 & McArthur et al., 2000 \\
\hline Runswick & R7A & 15.65 & 1.61 & 0.03 & McArthur et al., 2000 \\
\hline Saltwick Bay & Y06 43/7 & 15.63 & 1.53 & 0.03 & Li et al., 2012 \\
\hline Runswick & R10 & 15.62 & 1.58 & 0.03 & McArthur et al., 2000 \\
\hline Runswick & R5 & 15.60 & 1.91 & 0.02 & McArthur et al., 2000 \\
\hline Port Mulgrave & PM103 & 15.56 & 1.92 & 0.01 & McArthur et al., 2000 \\
\hline Port Mulgrave & PM20 & 15.53 & 1.84 & 0.04 & McArthur et al., 2000 \\
\hline Port Mulgrave & PM17 & 15.52 & 1.83 & 0.02 & McArthur et al., 2000 \\
\hline Port Mulgrave & PM15 & 15.51 & 1.36 & 0.09 & McArthur et al., 2000 \\
\hline Port Mulgrave & PM16 & 15.51 & 1.91 & 0.02 & McArthur et al., 2000 \\
\hline Port Mulgrave & PM13 & 15.50 & 1.99 & 0.06 & McArthur et al., 2000 \\
\hline Port Mulgrave & PM18 & 15.50 & 2.10 & 0.02 & McArthur et al., 2000 \\
\hline Port Mulgrave & PM7 & 15.47 & 1.76 & 0.05 & McArthur et al., 2000 \\
\hline Port Mulgrave & $\mathrm{PM} 2 \mathrm{C}$ & 15.45 & 2.12 & 0.03 & McArthur et al., 2000 \\
\hline Runswick & $\mathrm{R} 2 \mathrm{C}$ & 15.44 & 1.65 & 0.07 & McArthur et al., 2000 \\
\hline
\end{tabular}




\begin{tabular}{|c|c|c|c|c|c|}
\hline Port Mulgrave & PM8 & 15.40 & 1.66 & 0.03 & McArthur et al., 2000 \\
\hline Saltwick Bay & S1A & 15.38 & 2.03 & 0.03 & McArthur et al., 2000 \\
\hline Runswick & $\mathrm{R} 4 \mathrm{~B}$ & 15.34 & 1.69 & 0.01 & McArthur et al., 2000 \\
\hline Saltwick Bay & S2 & 15.33 & 2.06 & 0.04 & McArthur et al., 2000 \\
\hline Port Mulgrave & PM104 & 15.33 & 2.07 & 0.01 & McArthur et al., 2000 \\
\hline Port Mulgrave & PM3 & 15.25 & 2.14 & 0.03 & McArthur et al., 2000 \\
\hline Port Mulgrave & PM105 & 15.17 & 1.93 & 0.01 & McArthur et al., 2000 \\
\hline Port Mulgrave & PM111 & 15.16 & 1.91 & 0.00 & McArthur et al., 2000 \\
\hline Port Mulgrave & PM109 & 15.13 & 1.92 & 0.02 & McArthur et al., 2000 \\
\hline Port Mulgrave & PM21 & 15.06 & 1.54 & 0.13 & McArthur et al., 2000 \\
\hline Port Mulgrave & PM107 & 15.01 & 1.82 & 0.01 & McArthur et al., 2000 \\
\hline Port Mulgrave & PM106 & 14.98 & 1.50 & 0.02 & McArthur et al., 2000 \\
\hline Port Mulgrave & PM113 & 14.97 & 1.40 & 0.02 & McArthur et al., 2000 \\
\hline Port Mulgrave & PM112A & 14.96 & 1.55 & 0.02 & McArthur et al., 2000 \\
\hline Port Mulgrave & PM108 & 14.95 & 1.32 & 0.02 & McArthur et al., 2000 \\
\hline Kettleness & K117 & 14.92 & 1.33 & 0.01 & McArthur et al., 2000 \\
\hline Kettleness & K118A & 14.90 & 1.35 & 0.01 & McArthur et al., 2000 \\
\hline Port Mulgrave & PM101 & 14.86 & 1.57 & 0.01 & McArthur et al., 2000 \\
\hline Kettleness & K121 & 14.85 & 1.31 & 0.01 & McArthur et al., 2000 \\
\hline Robin Hood's Bay & HOOD 442 & 14.84 & 1.49 & 0.03 & Korte and Hesselbo, 2011 \\
\hline Robin Hood's Bay & HOOD 441 & 14.81 & 1.35 & 0.02 & Korte and Hesselbo, 2011 \\
\hline Port Mulgrave & PM102 & 14.81 & 1.20 & 0.03 & McArthur et al., 2000 \\
\hline Robin Hood's Bay & HOOD 440 & 14.81 & 1.51 & 0.03 & Korte and Hesselbo, 2011 \\
\hline Robin Hood's Bay & HOOD 439 & 14.80 & 1.49 & 0.03 & Korte and Hesselbo, 2011 \\
\hline Kettleness & K112B & 14.79 & 1.17 & 0.01 & McArthur et al., 2000 \\
\hline Kettleness & K111B & 14.69 & 1.24 & 0.01 & McArthur et al., 2000 \\
\hline Kettleness & $\mathrm{K} 111 \mathrm{C}$ & 14.69 & 1.26 & 0.01 & McArthur et al., 2000 \\
\hline Kettleness & K108B & 14.49 & 1.19 & 0.01 & McArthur et al., 2000 \\
\hline Robin Hood's Bay & HOOD 438 & 14.32 & 1.92 & 0.08 & Korte and Hesselbo, 2011 \\
\hline Robin Hood's Bay & HOOD 437 & 14.27 & 1.50 & 0.06 & Korte and Hesselbo, 2011 \\
\hline Kettleness & K105A & 14.25 & 1.23 & 0.01 & McArthur et al., 2000 \\
\hline Kettleness & K107 & 14.24 & 1.15 & 0.01 & McArthur et al., 2000 \\
\hline Staithes & St104 & 14.12 & 1.16 & 0.02 & McArthur et al., 2000 \\
\hline Hawsker Bay & HB7 & 14.07 & 1.10 & 0.00 & McArthur et al., 2000 \\
\hline Robin Hood's Bay & HOOD 434 & 14.04 & 1.50 & 0.04 & Korte and Hesselbo, 2011 \\
\hline Hawsker Bay & HB6 & 14.04 & 1.24 & 0.00 & McArthur et al., 2000 \\
\hline Hawsker Bay & HB3 & 14.03 & 1.67 & 0.00 & McArthur et al., 2000 \\
\hline Hawsker Bay & HB4 & 14.03 & 1.35 & 0.00 & McArthur et al., 2000 \\
\hline Hawsker Bay & HB2 & 14.02 & 1.20 & 0.00 & McArthur et al., 2000 \\
\hline Hawsker Bay & HB1 & 14.02 & 1.19 & 0.00 & McArthur et al., 2000 \\
\hline Robin Hood's Bay & HOOD 435 & 14.01 & 1.35 & 0.07 & Korte and Hesselbo, 2011 \\
\hline Robin Hood's Bay & HOOD 435 & 14.01 & 1.32 & 0.11 & Korte and Hesselbo, 2011 \\
\hline Robin Hood's Bay & HOOD 435 & 14.01 & 1.33 & 0.06 & Korte and Hesselbo, 2011 \\
\hline Robin Hood's Bay & HOOD $434 \mathrm{~B}$ & 14.01 & 1.12 & 0.02 & Korte and Hesselbo, 2011 \\
\hline Robin Hood's Bay & HOOD $434 \mathrm{~B}$ & 14.01 & 1.27 & 0.05 & Korte and Hesselbo, 2011 \\
\hline Robin Hood's Bay & HOOD 436 & 14.01 & 1.12 & 0.07 & Korte and Hesselbo, 2011 \\
\hline Robin Hood's Bay & HOOD 436 & 14.01 & 1.15 & 0.02 & Korte and Hesselbo, 2011 \\
\hline Robin Hood's Bay & HOOD 433 & 13.98 & 1.18 & 0.06 & Korte and Hesselbo, 2011 \\
\hline Staithes & St109 & 13.96 & 1.37 & 0.00 & McArthur et al., 2000 \\
\hline
\end{tabular}




\begin{tabular}{|c|c|c|c|c|c|}
\hline Hawsker Bay & HB11 & 13.95 & & 0.00 & McArthur et al., 2000 \\
\hline Robin Hood's Bay & HOOD 424 & 13.91 & 2.23 & 0.06 & Korte and Hesselbo, 2011 \\
\hline Robin Hood's Bay & HOOD 423 & 13.90 & 1.60 & 0.05 & Korte and Hesselbo, 2011 \\
\hline Hawsker Bay & HB8 & 13.78 & 1.15 & 0.00 & McArthur et al., 2000 \\
\hline Hawsker Bay & HB14 & 13.64 & 1.66 & 0.00 & McArthur et al., 2000 \\
\hline Hawsker Bay & HB12 & 13.63 & 1.85 & 0.00 & McArthur et al., 2000 \\
\hline Hawsker Bay & HB13 & 13.63 & 1.73 & 0.00 & McArthur et al., 2000 \\
\hline Hawsker Bay & HB15 & 13.50 & 1.75 & 0.00 & McArthur et al., 2000 \\
\hline Hawsker Bay & HB16 & 13.37 & 1.61 & 0.00 & McArthur et al., 2000 \\
\hline Robin Hood's Bay & HOOD 410 & 13.33 & 1.70 & 0.02 & Korte and Hesselbo, 2011 \\
\hline Hawsker Bay & HB17 & 13.13 & 1.72 & 0.00 & McArthur et al., 2000 \\
\hline Staithes & St116D & 13.07 & 1.62 & 0.05 & McArthur et al., 2000 \\
\hline Staithes & St102A & 13.06 & 1.29 & 0.02 & McArthur et al., 2000 \\
\hline Robin Hood's Bay & HOOD 400 B & 13.02 & 2.00 & 0.01 & Korte and Hesselbo, 2011 \\
\hline Robin Hood's Bay & HOOD 399 & 12.99 & 1.99 & 0.10 & Korte and Hesselbo, 2011 \\
\hline Staithes & St119B & 12.98 & 1.51 & 0.04 & McArthur et al., 2000 \\
\hline Robin Hood's Bay & HOOD 396 & 12.97 & 1.88 & 0.19 & Korte and Hesselbo, 2011 \\
\hline Staithes & St120 & 12.93 & 1.31 & 0.02 & McArthur et al., 2000 \\
\hline Staithes & St121 & 12.87 & 1.64 & 0.02 & McArthur et al., 2000 \\
\hline Staithes & St123 & 12.80 & 1.65 & 0.04 & McArthur et al., 2000 \\
\hline Staithes & St126B & 12.67 & 1.58 & 0.05 & McArthur et al., 2000 \\
\hline Robin Hood's Bay & HOOD 383 & 12.63 & 1.72 & 0.07 & Korte and Hesselbo, 2011 \\
\hline Robin Hood's Bay & HOOD 384 & 12.63 & 1.62 & 0.05 & Korte and Hesselbo, 2011 \\
\hline Robin Hood's Bay & HOOD 381 & 12.59 & 1.22 & 0.12 & Korte and Hesselbo, 2011 \\
\hline Staithes & St127A & 12.52 & 1.47 & 0.04 & McArthur et al., 2000 \\
\hline Staithes & St128A & 12.38 & 1.41 & 0.01 & McArthur et al., 2000 \\
\hline Robin Hood's Bay & HOOD 378 & 12.38 & 1.95 & 0.35 & Korte and Hesselbo, 2011 \\
\hline Robin Hood's Bay & HOOD 377 & 12.34 & 1.71 & 0.03 & Korte and Hesselbo, 2011 \\
\hline Robin Hood's Bay & HOOD $370 \mathrm{~A}$ & 12.32 & 1.60 & 0.02 & Korte and Hesselbo, 2011 \\
\hline Robin Hood's Bay & HOOD 365 & 12.30 & 1.67 & 0.02 & Korte and Hesselbo, 2011 \\
\hline Staithes & St129 & 12.30 & 1.72 & 0.01 & McArthur et al., 2000 \\
\hline Staithes & St130(1) & 12.30 & 1.73 & 0.01 & McArthur et al., 2000 \\
\hline Robin Hood's Bay & HOOD 364 & 12.28 & 1.41 & 0.02 & Korte and Hesselbo, 2011 \\
\hline Robin Hood's Bay & HOOD 363 & 12.28 & 1.67 & 0.15 & Korte and Hesselbo, 2011 \\
\hline Robin Hood's Bay & HOOD 356 & 12.27 & 1.50 & 0.05 & Korte and Hesselbo, 2011 \\
\hline Robin Hood's Bay & HOOD 354 & 12.25 & 1.77 & 0.06 & Korte and Hesselbo, 2011 \\
\hline Robin Hood's Bay & HOOD 348 & 12.22 & 1.99 & 0.10 & Korte and Hesselbo, 2011 \\
\hline Staithes & St131 & 12.18 & 1.58 & 0.04 & McArthur et al., 2000 \\
\hline Robin Hood's Bay & HOOD 342 & 12.10 & 1.62 & 0.06 & Korte and Hesselbo, 2011 \\
\hline Robin Hood's Bay & HOOD 339 & 12.07 & 1.83 & 0.11 & Korte and Hesselbo, 2011 \\
\hline Robin Hood's Bay & HOOD 334 & 12.06 & 2.04 & 0.29 & Korte and Hesselbo, 2011 \\
\hline Robin Hood's Bay & HOOD 303 & 12.00 & 1.28 & 0.03 & Korte and Hesselbo, 2011 \\
\hline Robin Hood's Bay & HOOD 304 & 12.00 & 1.35 & 0.03 & Korte and Hesselbo, 2011 \\
\hline Robin Hood's Bay & HOOD 259 & 11.86 & 1.78 & 0.05 & Korte and Hesselbo, 2011 \\
\hline Robin Hood's Bay & HOOD 277 & 11.41 & 1.39 & 0.02 & Korte and Hesselbo, 2011 \\
\hline Robin Hood's Bay & HOOD 281 & 11.31 & 1.75 & 0.01 & Korte and Hesselbo, 2011 \\
\hline Robin Hood's Bay & HOOD 283 & 11.26 & 2.03 & 0.43 & Korte and Hesselbo, 2011 \\
\hline Robin Hood's Bay & HOOD 284 & 11.25 & 1.52 & 0.04 & Korte and Hesselbo, 2011 \\
\hline Robin Hood's Bay & HOOD 285 & 11.21 & 1.04 & 0.20 & Korte and Hesselbo, 2011 \\
\hline
\end{tabular}




\begin{tabular}{|c|c|c|c|c|c|}
\hline Robin Hood's Bay & HOOD 286 & 11.17 & 1.34 & 0.03 & Korte and Hesselbo, 2011 \\
\hline Robin Hood's Bay & HOOD 291 & 11.11 & 1.03 & 0.20 & Korte and Hesselbo, 2011 \\
\hline Robin Hood's Bay & HOOD 298 & 10.99 & 1.41 & 0.19 & Korte and Hesselbo, 2011 \\
\hline Robin Hood's Bay & HOOD 299 & 10.99 & 1.34 & 0.08 & Korte and Hesselbo, 2011 \\
\hline Robin Hood's Bay & HOOD 313 & 10.96 & 1.41 & 0.04 & Korte and Hesselbo, 2011 \\
\hline Robin Hood's Bay & HOOD 314 & 10.95 & 1.42 & 0.29 & Korte and Hesselbo, 2011 \\
\hline Robin Hood's Bay & HOOD 315 & 10.94 & 1.46 & 0.11 & Korte and Hesselbo, 2011 \\
\hline Robin Hood's Bay & HOOD 318 & 10.92 & 1.89 & 0.34 & Korte and Hesselbo, 2011 \\
\hline Robin Hood's Bay & HOOD 323 & 10.86 & 1.50 & 0.02 & Korte and Hesselbo, 2011 \\
\hline Robin Hood's Bay & HOOD 321 & 10.85 & 0.90 & 0.05 & Korte and Hesselbo, 2011 \\
\hline Robin Hood's Bay & HOOD 466 & 10.79 & 1.66 & 0.07 & Korte and Hesselbo, 2011 \\
\hline Robin Hood's Bay & HOOD 470 & 10.78 & 1.29 & 0.04 & Korte and Hesselbo, 2011 \\
\hline Robin Hood's Bay & HOOD 469 & 10.77 & 1.79 & 0.02 & Korte and Hesselbo, 2011 \\
\hline Robin Hood's Bay & HOOD 462 & 10.76 & 1.78 & 0.10 & Korte and Hesselbo, 2011 \\
\hline Robin Hood's Bay & HOOD 465 & 10.75 & 1.01 & 0.02 & Korte and Hesselbo, 2011 \\
\hline Robin Hood's Bay & HOOD 472 & 10.74 & 0.60 & 0.13 & Korte and Hesselbo, 2011 \\
\hline Robin Hood's Bay & HOOD 473 & 10.74 & 1.70 & 0.16 & Korte and Hesselbo, 2011 \\
\hline Robin Hood's Bay & HOOD 474 & 10.74 & 1.16 & 0.02 & Korte and Hesselbo, 2011 \\
\hline Robin Hood's Bay & HOOD 453 & 10.68 & 0.88 & 0.30 & Korte and Hesselbo, 2011 \\
\hline Robin Hood's Bay & HOOD 451 & 10.68 & 0.99 & 0.18 & Korte and Hesselbo, 2011 \\
\hline Robin Hood's Bay & HOOD 452 & 10.68 & 1.06 & 0.42 & Korte and Hesselbo, 2011 \\
\hline Robin Hood's Bay & HOOD 450 & 10.67 & 1.03 & 0.02 & Korte and Hesselbo, 2011 \\
\hline Robin Hood's Bay & HOOD 052 & 10.60 & 1.15 & 0.23 & Korte and Hesselbo, 2011 \\
\hline Robin Hood's Bay & HOOD 051 & 10.59 & 1.18 & 0.07 & Korte and Hesselbo, 2011 \\
\hline Robin Hood's Bay & HOOD 454 & 10.59 & 2.06 & 0.02 & Korte and Hesselbo, 2011 \\
\hline Robin Hood's Bay & HOOD 458 & 10.54 & 1.41 & 0.16 & Korte and Hesselbo, 2011 \\
\hline Robin Hood's Bay & HOOD 053 & 10.50 & 1.38 & 0.07 & Korte and Hesselbo, 2011 \\
\hline Robin Hood's Bay & HOOD $050 \mathrm{~A}$ & 10.45 & 0.95 & 0.07 & Korte and Hesselbo, 2011 \\
\hline Robin Hood's Bay & HOOD 459 & 10.45 & 0.95 & 0.01 & Korte and Hesselbo, 2011 \\
\hline Robin Hood's Bay & HOOD 042 B & 10.36 & 1.36 & 0.06 & Korte and Hesselbo, 2011 \\
\hline Robin Hood's Bay & HOOD 044 B & 10.29 & 1.29 & 0.08 & Korte and Hesselbo, 2011 \\
\hline Robin Hood's Bay & HOOD 037 A & 10.24 & 2.06 & 0.15 & Korte and Hesselbo, 2011 \\
\hline Robin Hood's Bay & HOOD 036 B & 10.22 & 1.50 & 0.33 & Korte and Hesselbo, 2011 \\
\hline Robin Hood's Bay & HOOD $035 \mathrm{C}$ & 10.16 & 2.12 & 0.12 & Korte and Hesselbo, 2011 \\
\hline Robin Hood's Bay & HOOD 067 & 9.73 & 1.39 & 0.07 & Korte and Hesselbo, 2011 \\
\hline Robin Hood's Bay & HOOD 064 & 9.72 & 1.18 & 0.14 & Korte and Hesselbo, 2011 \\
\hline Robin Hood's Bay & HOOD 228 & 9.72 & 2.05 & 0.19 & Korte and Hesselbo, 2011 \\
\hline Robin Hood's Bay & HOOD 226 & 9.71 & 1.77 & 0.27 & Korte and Hesselbo, 2011 \\
\hline Robin Hood's Bay & HOOD 062 & 9.70 & 1.35 & 0.04 & Korte and Hesselbo, 2011 \\
\hline Robin Hood's Bay & HOOD 056 & 9.67 & 1.37 & 0.12 & Korte and Hesselbo, 2011 \\
\hline Robin Hood's Bay & HOOD 229 & 9.59 & 1.95 & 0.35 & Korte and Hesselbo, 2011 \\
\hline Robin Hood's Bay & HOOD 232 & 9.55 & 1.68 & 0.16 & Korte and Hesselbo, 2011 \\
\hline Robin Hood's Bay & HOOD 218 & 9.46 & 1.63 & 0.20 & Korte and Hesselbo, 2011 \\
\hline Robin Hood's Bay & HOOD 212 & 9.41 & 1.32 & 0.12 & Korte and Hesselbo, 2011 \\
\hline Robin Hood's Bay & HOOD 214 & 9.41 & 1.16 & 0.41 & Korte and Hesselbo, 2011 \\
\hline Robin Hood's Bay & HOOD 220 & 9.39 & 1.99 & 0.05 & Korte and Hesselbo, 2011 \\
\hline Robin Hood's Bay & HOOD 209 & 9.36 & 1.08 & 0.02 & Korte and Hesselbo, 2011 \\
\hline Robin Hood's Bay & HOOD 208 & 9.27 & 0.95 & 0.05 & Korte and Hesselbo, 2011 \\
\hline Robin Hood's Bay & HOOD 200 & 9.26 & 1.31 & 0.07 & Korte and Hesselbo, 2011 \\
\hline
\end{tabular}




\begin{tabular}{|c|c|c|c|c|c|}
\hline Robin Hood's Bay & HOOD 205 & 9.24 & 1.16 & 0.16 & Korte and Hesselbo, 2011 \\
\hline Robin Hood's Bay & HOOD 239 & 9.08 & 1.41 & 0.30 & Korte and Hesselbo, 2011 \\
\hline Robin Hood's Bay & HOOD 110 & 9.04 & 1.56 & 0.28 & Korte and Hesselbo, 2011 \\
\hline Robin Hood's Bay & HOOD $111 \mathrm{C}$ & 9.02 & 1.73 & 0.09 & Korte and Hesselbo, 2011 \\
\hline Robin Hood's Bay & HOOD $106 \mathrm{~B}$ & 9.01 & 1.02 & 0.23 & Korte and Hesselbo, 2011 \\
\hline Robin Hood's Bay & HOOD 106 B & 9.01 & 1.51 & 0.13 & Korte and Hesselbo, 2011 \\
\hline Robin Hood's Bay & HOOD 106 B & 9.01 & 1.04 & 0.02 & Korte and Hesselbo, 2011 \\
\hline Robin Hood's Bay & HOOD 106 B & 9.01 & 1.74 & 0.18 & Korte and Hesselbo, 2011 \\
\hline Robin Hood's Bay & HOOD $106 \mathrm{C}$ & 9.01 & 1.59 & 0.08 & Korte and Hesselbo, 2011 \\
\hline Robin Hood's Bay & HOOD $106 \mathrm{C}$ & 9.01 & 1.44 & 0.03 & Korte and Hesselbo, 2011 \\
\hline Robin Hood's Bay & HOOD $106 \mathrm{C}$ & 9.01 & 1.06 & 0.02 & Korte and Hesselbo, 2011 \\
\hline Robin Hood's Bay & HOOD 109 & 9.01 & 1.46 & 0.06 & Korte and Hesselbo, 2011 \\
\hline Robin Hood's Bay & HOOD 108 & 9.01 & 1.34 & 0.06 & Korte and Hesselbo, 2011 \\
\hline Robin Hood's Bay & HOOD $102 \mathrm{C}$ & 9.00 & 1.65 & 0.07 & Korte and Hesselbo, 2011 \\
\hline Robin Hood's Bay & HOOD 101 & 8.99 & 1.76 & 0.08 & Korte and Hesselbo, 2011 \\
\hline Robin Hood's Bay & HOOD 100 & 8.98 & 1.91 & 0.11 & Korte and Hesselbo, 2011 \\
\hline Robin Hood's Bay & HOOD 097 B & 8.97 & 1.43 & 0.07 & Korte and Hesselbo, 2011 \\
\hline Robin Hood's Bay & HOOD $097 \mathrm{C}$ & 8.97 & 2.04 & 0.08 & Korte and Hesselbo, 2011 \\
\hline Robin Hood's Bay & HOOD 093 & 8.78 & 1.74 & 0.40 & Korte and Hesselbo, 2011 \\
\hline Robin Hood's Bay & HOOD 089 B & 8.77 & 1.59 & 0.07 & Korte and Hesselbo, 2011 \\
\hline Robin Hood's Bay & HOOD 094 & 8.77 & 1.75 & 0.20 & Korte and Hesselbo, 2011 \\
\hline Robin Hood's Bay & HOOD 095 & 8.74 & 1.53 & 0.12 & Korte and Hesselbo, 2011 \\
\hline Robin Hood's Bay & HOOD 088 & 8.67 & 1.59 & 0.04 & Korte and Hesselbo, 2011 \\
\hline Robin Hood's Bay & HOOD 080 & 8.31 & 1.94 & 0.23 & Korte and Hesselbo, 2011 \\
\hline Robin Hood's Bay & HOOD 081 & 8.29 & 2.14 & 0.26 & Korte and Hesselbo, 2011 \\
\hline Robin Hood's Bay & HOOD 078 A & 8.26 & 1.97 & 0.35 & Korte and Hesselbo, 2011 \\
\hline Robin Hood's Bay & HOOD 029 A & 7.77 & 1.65 & 0.19 & Korte and Hesselbo, 2011 \\
\hline Robin Hood's Bay & HOOD 028 A & 7.73 & 1.53 & 0.22 & Korte and Hesselbo, 2011 \\
\hline Robin Hood's Bay & HOOD 021 & 6.95 & 2.17 & 0.07 & Korte and Hesselbo, 2011 \\
\hline Robin Hood's Bay & HOOD 020 & 6.85 & 1.88 & 0.03 & Korte and Hesselbo, 2011 \\
\hline Robin Hood's Bay & HOOD 019 & 6.79 & 1.80 & 0.19 & Korte and Hesselbo, 2011 \\
\hline Robin Hood's Bay & HOOD 014 A & 6.63 & 1.59 & 0.06 & Korte and Hesselbo, 2011 \\
\hline Robin Hood's Bay & HOOD $013 \mathrm{H}$ & 6.54 & 1.67 & 0.15 & Korte and Hesselbo, 2011 \\
\hline Robin Hood's Bay & HOOD 001 & 5.56 & 2.43 & 0.10 & Korte and Hesselbo, 2011 \\
\hline Hallaig Waterfall & Hal_17 & 5.24 & 2.15 & 0.08 & present study \\
\hline
\end{tabular}




\begin{tabular}{|c|c|c|c|c|c|}
\hline section & sample No & $\begin{array}{l}\text { Zone } \\
\text { No. }\end{array}$ & $\begin{array}{c}\mathrm{Sr} / \mathrm{Ca} \\
\mathrm{mmol} / \mathrm{mol}\end{array}$ & $\begin{array}{c}\mathrm{Mn} / \mathrm{Ca} \\
\mathrm{mmol} / \mathrm{mol}\end{array}$ & source \\
\hline Robin Hood's Bay & HOOD 406 & 13.12 & 0.436 & 0.07 & Korte and Hesselbo 2011 \\
\hline Robin Hood's Bay & HOOD 404 A & 13.08 & 0.468 & 0.03 & Korte and Hesselbo 2011 \\
\hline Robin Hood's Bay & HOOD $400 \mathrm{C}$ & 13.02 & 0.499 & 0.11 & Korte and Hesselbo 2011 \\
\hline Robin Hood's Bay & HOOD 394 & 12.97 & 0.836 & 0.11 & Korte and Hesselbo 2011 \\
\hline Robin Hood's Bay & HOOD 382 & 12.63 & 0.640 & 0.21 & Korte and Hesselbo 2011 \\
\hline Robin Hood's Bay & HOOD 370 & 12.32 & 0.504 & 0.13 & Korte and Hesselbo 2011 \\
\hline Robin Hood's Bay & HOOD 358 & 12.27 & 0.542 & 0.12 & Korte and Hesselbo 2011 \\
\hline Robin Hood's Bay & HOOD 353 & 12.24 & 0.400 & 0.15 & Korte and Hesselbo 2011 \\
\hline Robin Hood's Bay & HOOD 352 & 12.24 & 0.440 & 0.14 & Korte and Hesselbo 2011 \\
\hline Robin Hood's Bay & HOOD 350 & 12.24 & 0.502 & 0.20 & Korte and Hesselbo 2011 \\
\hline Robin Hood's Bay & HOOD 345 A & 12.12 & 0.410 & 0.18 & Korte and Hesselbo 2011 \\
\hline Robin Hood's Bay & HOOD 338 & 12.07 & 0.505 & 0.11 & Korte and Hesselbo 2011 \\
\hline Robin Hood's Bay & HOOD 334 & 12.06 & 0.499 & 0.10 & Korte and Hesselbo 2011 \\
\hline Robin Hood's Bay & HOOD 333 & 12.04 & 0.454 & 0.18 & Korte and Hesselbo 2011 \\
\hline Robin Hood's Bay & HOOD 305 & 12.00 & 0.474 & 0.10 & Korte and Hesselbo 2011 \\
\hline Robin Hood's Bay & HOOD 302 & 12.00 & 0.537 & 0.10 & Korte and Hesselbo 2011 \\
\hline Robin Hood's Bay & HOOD 305 & 12.00 & 0.375 & 0.24 & Korte and Hesselbo 2011 \\
\hline Robin Hood's Bay & HOOD 301 & 12.00 & 0.379 & 0.10 & Korte and Hesselbo 2011 \\
\hline Robin Hood's Bay & HOOD 246 & 11.98 & 0.539 & 0.17 & Korte and Hesselbo 2011 \\
\hline Robin Hood's Bay & HOOD 248 & 11.91 & 0.497 & 0.30 & Korte and Hesselbo 2011 \\
\hline Robin Hood's Bay & HOOD 300 & 11.89 & 0.667 & 0.16 & Korte and Hesselbo 2011 \\
\hline Robin Hood's Bay & HOOD 300 & 11.89 & 0.530 & 0.14 & Korte and Hesselbo 2011 \\
\hline Robin Hood's Bay & HOOD 251 & 11.89 & 0.459 & 0.24 & Korte and Hesselbo 2011 \\
\hline Robin Hood's Bay & HOOD 260 & 11.86 & 0.509 & 0.15 & Korte and Hesselbo 2011 \\
\hline Robin Hood's Bay & HOOD 255 & 11.85 & 0.553 & 0.16 & Korte and Hesselbo 2011 \\
\hline Robin Hood's Bay & HOOD 256 & 11.85 & 0.442 & 0.16 & Korte and Hesselbo 2011 \\
\hline Robin Hood's Bay & HOOD 256 & 11.85 & 0.330 & 0.17 & Korte and Hesselbo 2011 \\
\hline Robin Hood's Bay & HOOD 254 & 11.85 & 0.388 & 0.04 & Korte and Hesselbo 2011 \\
\hline Robin Hood's Bay & HOOD 254 & 11.85 & 0.416 & 0.04 & Korte and Hesselbo 2011 \\
\hline Robin Hood's Bay & HOOD 261 & 11.82 & 0.372 & 0.08 & Korte and Hesselbo 2011 \\
\hline Robin Hood's Bay & HOOD 331 A & 11.73 & 0.440 & 0.20 & Korte and Hesselbo 2011 \\
\hline Robin Hood's Bay & HOOD 271 & 11.71 & 0.537 & 0.21 & Korte and Hesselbo 2011 \\
\hline Robin Hood's Bay & HOOD 270 & 11.67 & 0.501 & 0.40 & Korte and Hesselbo 2011 \\
\hline Robin Hood's Bay & HOOD 279 & 11.47 & 0.437 & 0.07 & Korte and Hesselbo 2011 \\
\hline Robin Hood's Bay & HOOD 275 & 11.43 & 0.477 & 0.16 & Korte and Hesselbo 2011 \\
\hline Robin Hood's Bay & HOOD 276 B & 11.41 & 0.597 & 0.34 & Korte and Hesselbo 2011 \\
\hline Robin Hood's Bay & HOOD 276 A & 11.41 & 0.458 & 0.14 & Korte and Hesselbo 2011 \\
\hline Robin Hood's Bay & HOOD 274 B & 11.35 & 0.512 & 0.23 & Korte and Hesselbo 2011 \\
\hline Robin Hood's Bay & HOOD 273 & 11.33 & 0.530 & 0.23 & Korte and Hesselbo 2011 \\
\hline Robin Hood's Bay & HOOD 273 & 11.33 & 0.558 & 0.21 & Korte and Hesselbo 2011 \\
\hline Robin Hood's Bay & HOOD 282 & 11.31 & 0.648 & 0.19 & Korte and Hesselbo 2011 \\
\hline Robin Hood's Bay & HOOD 280 & 11.30 & 0.487 & 0.28 & Korte and Hesselbo 2011 \\
\hline Robin Hood's Bay & HOOD 050 A & 10.45 & 0.363 & 0.15 & Korte and Hesselbo 2011 \\
\hline Robin Hood's Bay & HOOD $050 \mathrm{~A}$ & 10.45 & 0.359 & 0.16 & Korte and Hesselbo 2011 \\
\hline Robin Hood's Bay & HOOD $050 \mathrm{C}$ & 10.45 & 0.357 & 0.09 & Korte and Hesselbo 2011 \\
\hline Robin Hood's Bay & HOOD 046 & 10.40 & 0.394 & 0.08 & Korte and Hesselbo 2011 \\
\hline
\end{tabular}


Robin Hood's Bay

Robin Hood's Bay

Robin Hood's Bay

Robin Hood's Bay

Robin Hood's Bay

Robin Hood's Bay

Robin Hood's Bay

Robin Hood's Bay

Robin Hood's Bay

Robin Hood's Bay

Robin Hood's Bay

Robin Hood's Bay

Robin Hood's Bay

Robin Hood's Bay

Robin Hood's Bay

Robin Hood's Bay

Robin Hood's Bay

Robin Hood's Bay

Robin Hood's Bay

Robin Hood's Bay

Robin Hood's Bay

Robin Hood's Bay

Robin Hood's Bay

Robin Hood's Bay

Robin Hood's Bay

Robin Hood's Bay

Robin Hood's Bay

Robin Hood's Bay

Robin Hood's Bay

Robin Hood's Bay

Robin Hood's Bay

Robin Hood's Bay

Robin Hood's Bay

Robin Hood's Bay

Robin Hood's Bay

Robin Hood's Bay

Robin Hood's Bay

Robin Hood's Bay

Robin Hood's Bay

Robin Hood's Bay

Robin Hood's Bay

Robin Hood's Bay

Robin Hood's Bay

Robin Hood's Bay

Robin Hood's Bay

Robin Hood's Bay

Robin Hood's Bay

Robin Hood's Bay

Robin Hood's Bay

\begin{tabular}{|c|c|c|c|c|}
\hline HOOD 046 & 10.40 & 0.418 & 0.09 & Korte and Hesselbo 2011 \\
\hline HOOD $042 \mathrm{C}$ & 10.36 & 0.466 & 0.16 & Korte and Hesselbo 2011 \\
\hline HOOD $042 \mathrm{C}$ & 10.36 & 0.410 & 0.09 & Korte and Hesselbo 2011 \\
\hline HOOD $039 \mathrm{~B}$ & 10.31 & 0.444 & 0.34 & Korte and Hesselbo 2011 \\
\hline HOOD 044 B & 10.29 & 0.424 & 0.19 & Korte and Hesselbo 2011 \\
\hline HOOD 044 B & 10.29 & 0.417 & 0.07 & Korte and Hesselbo 2011 \\
\hline HOOD $036 \mathrm{~B}$ & 10.22 & 0.509 & 0.12 & Korte and Hesselbo 2011 \\
\hline HOOD 035 B & 10.16 & 0.584 & 0.14 & Korte and Hesselbo 2011 \\
\hline HOOD 035 B & 10.16 & 0.583 & 0.09 & Korte and Hesselbo 2011 \\
\hline HOOD 035 A & 10.16 & 0.552 & 0.14 & Korte and Hesselbo 2011 \\
\hline HOOD 067 & 9.73 & 0.412 & 0.16 & Korte and Hesselbo 2011 \\
\hline HOOD 067 & 9.73 & 0.420 & 0.04 & Korte and Hesselbo 2011 \\
\hline HOOD 063 A & 9.73 & 0.412 & 0.11 & Korte and Hesselbo 2011 \\
\hline HOOD $066 \mathrm{~B}$ & 9.72 & 0.388 & 0.05 & Korte and Hesselbo 2011 \\
\hline HOOD 064 & 9.72 & 0.371 & 0.06 & Korte and Hesselbo 2011 \\
\hline HOOD 066 & 9.72 & 0.447 & 0.04 & Korte and Hesselbo 2011 \\
\hline HOOD 066 & 9.72 & 0.379 & 0.04 & Korte and Hesselbo 2011 \\
\hline HOOD 065 & 9.72 & 0.398 & 0.09 & Korte and Hesselbo 2011 \\
\hline HOOD 228 & 9.72 & 0.506 & 0.25 & Korte and Hesselbo 2011 \\
\hline HOOD 228 & 9.72 & 0.506 & 0.24 & Korte and Hesselbo 2011 \\
\hline HOOD 227 & 9.72 & 0.545 & 0.11 & Korte and Hesselbo 2011 \\
\hline HOOD 227 & 9.72 & 0.561 & 0.16 & Korte and Hesselbo 2011 \\
\hline HOOD 227 & 9.72 & 0.481 & 0.31 & Korte and Hesselbo 2011 \\
\hline HOOD 225 & 9.71 & 0.573 & 0.33 & Korte and Hesselbo 2011 \\
\hline HOOD 225 & 9.71 & 0.560 & 0.14 & Korte and Hesselbo 2011 \\
\hline HOOD 057 & 9.67 & 0.400 & 0.09 & Korte and Hesselbo 2011 \\
\hline HOOD 058 & 9.67 & 0.416 & 0.18 & Korte and Hesselbo 2011 \\
\hline HOOD $055 \mathrm{~A}$ & 9.67 & 0.389 & 0.14 & Korte and Hesselbo 2011 \\
\hline HOOD $330 \mathrm{~B}$ & 9.61 & 0.474 & 0.03 & Korte and Hesselbo 2011 \\
\hline HOOD 232 & 9.55 & 0.567 & 0.17 & Korte and Hesselbo 2011 \\
\hline HOOD 223 & 9.42 & 0.472 & 0.45 & Korte and Hesselbo 2011 \\
\hline HOOD 222 & 9.42 & 0.714 & 0.22 & Korte and Hesselbo 2011 \\
\hline HOOD 214 & 9.41 & 0.463 & 0.20 & Korte and Hesselbo 2011 \\
\hline HOOD 213 & 9.41 & 0.649 & 0.19 & Korte and Hesselbo 2011 \\
\hline HOOD 213 & 9.41 & 0.593 & 0.29 & Korte and Hesselbo 2011 \\
\hline HOOD 214 & 9.41 & 0.423 & 0.07 & Korte and Hesselbo 2011 \\
\hline HOOD 210 & 9.36 & 0.387 & 0.05 & Korte and Hesselbo 2011 \\
\hline HOOD 206 & 9.27 & 0.383 & 0.08 & Korte and Hesselbo 2011 \\
\hline HOOD 206 & 9.27 & 0.395 & 0.10 & Korte and Hesselbo 2011 \\
\hline HOOD 243 & 9.13 & 0.665 & 0.19 & Korte and Hesselbo 2011 \\
\hline HOOD 243 & 9.13 & 0.555 & 0.14 & Korte and Hesselbo 2011 \\
\hline HOOD 243 & 9.13 & 0.576 & 0.24 & Korte and Hesselbo 2011 \\
\hline HOOD 243 & 9.13 & 0.515 & 0.44 & Korte and Hesselbo 2011 \\
\hline HOOD 236 & 9.12 & 0.610 & 0.10 & Korte and Hesselbo 2011 \\
\hline HOOD 236 & 9.12 & 0.587 & 0.27 & Korte and Hesselbo 2011 \\
\hline HOOD 236 & 9.12 & 0.583 & 0.09 & Korte and Hesselbo 2011 \\
\hline HOOD 237 & 9.12 & 0.590 & 0.11 & Korte and Hesselbo 2011 \\
\hline HOOD 241 & 9.08 & 0.572 & 0.12 & Korte and Hesselbo 2011 \\
\hline HOOD 240 & 9.08 & 0.402 & 0.02 & Korte and Hesselbo 2011 \\
\hline
\end{tabular}


Robin Hood's Bay

Robin Hood's Bay

Robin Hood's Bay

Robin Hood's Bay

Robin Hood's Bay

Robin Hood's Bay

Robin Hood's Bay

Robin Hood's Bay

Robin Hood's Bay

Robin Hood's Bay

Robin Hood's Bay

Robin Hood's Bay

Robin Hood's Bay

Robin Hood's Bay

Robin Hood's Bay

Robin Hood's Bay

Robin Hood's Bay

Robin Hood's Bay

Robin Hood's Bay

Robin Hood's Bay

Robin Hood's Bay

Robin Hood's Bay

Robin Hood's Bay

Robin Hood's Bay

Robin Hood's Bay

Robin Hood's Bay

Robin Hood's Bay

Robin Hood's Bay

Robin Hood's Bay

Robin Hood's Bay

Robin Hood's Bay

Robin Hood's Bay

Robin Hood's Bay

Robin Hood's Bay

Robin Hood's Bay

Robin Hood's Bay

Robin Hood's Bay

Robin Hood's Bay

Robin Hood's Bay

Robin Hood's Bay

Robin Hood's Bay

Robin Hood's Bay

Robin Hood's Bay

Robin Hood's Bay

Robin Hood's Bay

Robin Hood's Bay

Robin Hood's Bay

Robin Hood's Bay

Robin Hood's Bay

\begin{tabular}{|c|c|c|c|}
\hline HOOD 238 & 9.06 & 0.548 & 0.07 \\
\hline HOOD $111 \mathrm{~A}$ & 9.02 & 0.476 & 0.06 \\
\hline HOOD $111 \mathrm{~A}$ & 9.02 & 0.484 & 0.03 \\
\hline HOOD 111 & 9.02 & 0.511 & 0.08 \\
\hline HOOD 111 & 9.02 & 0.526 & 0.21 \\
\hline HOOD 111 & 9.02 & 0.508 & 0.12 \\
\hline HOOD 103 D & 9.00 & 0.488 & 0.07 \\
\hline HOOD 103 D & 9.00 & 0.470 & 0.09 \\
\hline HOOD 103 B & 9.00 & 0.607 & 0.10 \\
\hline HOOD 103 B & 9.00 & 0.566 & 0.10 \\
\hline HOOD 103 A & 9.00 & 0.584 & 0.09 \\
\hline HOOD 103 A & 9.00 & 0.561 & 0.10 \\
\hline HOOD 103 A & 9.00 & 0.575 & 0.19 \\
\hline HOOD 101 & 8.99 & 0.635 & 0.17 \\
\hline HOOD 101 & 8.99 & 0.621 & 0.20 \\
\hline HOOD 101 & 8.99 & 0.557 & 0.17 \\
\hline HOOD 099 & 8.98 & 0.568 & 0.18 \\
\hline HOOD 098 & 8.98 & 0.612 & 0.12 \\
\hline HOOD 098 & 8.98 & 0.515 & 0.18 \\
\hline HOOD 098 & 8.98 & 0.634 & 0.20 \\
\hline HOOD $097 \mathrm{C}$ & 8.97 & 0.525 & 0.35 \\
\hline HOOD 097 A & 8.97 & 0.492 & 0.10 \\
\hline HOOD 097 A & 8.97 & 0.516 & 0.10 \\
\hline HOOD 097 A & 8.97 & 0.507 & 0.15 \\
\hline HOOD 096 & 8.95 & 0.610 & 0.09 \\
\hline HOOD 096 & 8.95 & 0.597 & 0.08 \\
\hline HOOD 096 & 8.95 & 0.616 & 0.06 \\
\hline HOOD $091 \mathrm{~A}$ & 8.78 & 0.547 & 0.34 \\
\hline HOOD 095 & 8.74 & 0.555 & 0.27 \\
\hline HOOD 087 A & 8.63 & 0.540 & 0.15 \\
\hline HOOD 086 D & 8.54 & 0.543 & 0.16 \\
\hline HOOD 086 A & 8.54 & 0.485 & 0.08 \\
\hline HOOD $077 \mathrm{~A}$ & 8.25 & 0.605 & 0.23 \\
\hline HOOD 076 A & 8.22 & 0.571 & 0.14 \\
\hline HOOD 033 A & 8.21 & 0.539 & 0.22 \\
\hline HOOD 033 A & 8.21 & 0.618 & 0.33 \\
\hline HOOD $034 \mathrm{~A}$ & 8.17 & 0.726 & 0.45 \\
\hline HOOD $034 \mathrm{~A}$ & 8.17 & 0.643 & 0.42 \\
\hline HOOD 032 B & 8.08 & 0.566 & 0.12 \\
\hline HOOD 032 B & 8.08 & 0.548 & 0.12 \\
\hline HOOD 032 A & 8.08 & 0.582 & 0.27 \\
\hline HOOD $032 \mathrm{~A}$ & 8.08 & 0.620 & 0.37 \\
\hline HOOD 031 & 7.84 & 0.660 & 0.25 \\
\hline HOOD $031 \mathrm{~A}$ & 7.84 & 0.427 & 0.16 \\
\hline HOOD $031 \mathrm{~A}$ & 7.84 & 0.416 & 0.13 \\
\hline HOOD 029 B & 7.77 & 0.651 & 0.18 \\
\hline HOOD 023 & 7.47 & 0.534 & 0.13 \\
\hline HOOD 023 & 7.47 & 0.492 & 0.09 \\
\hline HOOD 017 A & 7.07 & 0.676 & 0.29 \\
\hline
\end{tabular}

Korte and Hesselbo 2011

Korte and Hesselbo 2011

Korte and Hesselbo 2011

Korte and Hesselbo 2011

Korte and Hesselbo 2011

Korte and Hesselbo 2011

Korte and Hesselbo 2011

Korte and Hesselbo 2011

Korte and Hesselbo 2011

Korte and Hesselbo 2011

Korte and Hesselbo 2011

Korte and Hesselbo 2011

Korte and Hesselbo 2011

Korte and Hesselbo 2011

Korte and Hesselbo 2011

Korte and Hesselbo 2011

Korte and Hesselbo 2011

Korte and Hesselbo 2011

Korte and Hesselbo 2011

Korte and Hesselbo 2011

Korte and Hesselbo 2011

Korte and Hesselbo 2011

Korte and Hesselbo 2011

Korte and Hesselbo 2011

Korte and Hesselbo 2011

Korte and Hesselbo 2011

Korte and Hesselbo 2011

Korte and Hesselbo 2011

Korte and Hesselbo 2011

Korte and Hesselbo 2011

Korte and Hesselbo 2011

Korte and Hesselbo 2011

Korte and Hesselbo 2011

Korte and Hesselbo 2011

Korte and Hesselbo 2011

Korte and Hesselbo 2011

Korte and Hesselbo 2011

Korte and Hesselbo 2011

Korte and Hesselbo 2011

Korte and Hesselbo 2011

Korte and Hesselbo 2011

Korte and Hesselbo 2011

Korte and Hesselbo 2011

Korte and Hesselbo 2011

Korte and Hesselbo 2011

Korte and Hesselbo 2011

Korte and Hesselbo 2011

Korte and Hesselbo 2011

Korte and Hesselbo 2011 


\begin{tabular}{|c|c|c|c|c|c|}
\hline Robin Hood's Bay & HOOD 018 B & 6.99 & 0.560 & 0.09 & Korte and Hesselbo 2011 \\
\hline Robin Hood's Bay & HOOD $011 \mathrm{~B}$ & 6.43 & 0.596 & 0.10 & Korte and Hesselbo 2011 \\
\hline Robin Hood's Bay & HOOD 011 A & 6.43 & 0.493 & 0.14 & Korte and Hesselbo 2011 \\
\hline Robin Hood's Bay & HOOD $010 \mathrm{C}$ & 5.87 & 0.621 & 0.35 & Korte and Hesselbo 2011 \\
\hline Robin Hood's Bay & HOOD 010 B & 5.87 & 0.534 & 0.16 & Korte and Hesselbo 2011 \\
\hline Robin Hood's Bay & HOOD 010 B & 5.87 & 0.552 & 0.31 & Korte and Hesselbo 2011 \\
\hline Robin Hood's Bay & HOOD 010 A & 5.87 & 0.549 & 0.19 & Korte and Hesselbo 2011 \\
\hline Hallaig Waterfall & Hal_5 & 5.70 & 0.832 & 0.20 & present study \\
\hline Hallaig Waterfall & Hal_5 & 5.70 & 0.777 & 0.13 & present study \\
\hline Hallaig Waterfall & Hal_3 & 5.67 & 0.668 & 0.31 & present study \\
\hline Robin Hood's Bay & HOOD 007 & 5.57 & 0.657 & 0.21 & Korte and Hesselbo 2011 \\
\hline Robin Hood's Bay & HOOD 002 & 5.49 & 0.595 & 0.14 & Korte and Hesselbo 2011 \\
\hline Hallaig Waterfall & Hal_10 & 5.35 & 0.703 & 0.16 & present study \\
\hline Hallaig Waterfall & Hal_10 & 5.35 & 0.682 & 0.14 & present study \\
\hline Hallaig Waterfall & Hal_10 & 5.35 & 0.670 & 0.09 & present study \\
\hline Hallaig Waterfall & Hal_12 & 5.32 & 0.720 & 0.20 & present study \\
\hline Hallaig Waterfall & Hal_12 & 5.32 & 0.763 & 0.17 & present study \\
\hline Hallaig Waterfall & Hal_16 & 5.25 & 0.642 & 0.10 & present study \\
\hline Hallaig Waterfall & Hal_16 & 5.25 & 0.620 & 0.05 & present study \\
\hline Hallaig Waterfall & Hal_36 & 5.21 & 0.709 & 0.28 & present study \\
\hline Hallaig Waterfall & Hal_19 & 5.21 & 0.631 & 0.28 & present study \\
\hline Hallaig Waterfall & Hal_36 & 5.21 & 0.704 & 0.21 & present study \\
\hline Hallaig Waterfall & Hal_19 & 5.21 & 0.715 & 0.09 & present study \\
\hline Hallaig Waterfall & Hal_34 & 5.20 & 0.674 & 0.31 & present study \\
\hline Hallaig Waterfall & Hal_35 & 5.20 & 0.699 & 0.23 & present study \\
\hline Hallaig Waterfall & Hal_32 & 5.18 & 0.648 & 0.15 & present study \\
\hline Hallaig Waterfall & Hal_32 & 5.18 & 0.602 & 0.02 & present study \\
\hline Hallaig Waterfall & Hal_31 & 5.17 & 0.627 & 0.06 & present study \\
\hline Hallaig Waterfall & Hal_31 & 5.17 & 0.617 & 0.06 & present study \\
\hline Hallaig Waterfall & Hal_30 & 5.17 & 0.673 & 0.31 & present study \\
\hline Hallaig Waterfall & Hal_21 & 5.13 & 0.592 & 0.03 & present study \\
\hline Hallaig Waterfall & Hal_21 & 5.13 & 0.578 & 0.02 & present study \\
\hline Hallaig Waterfall & Hal_22 & 5.13 & 0.651 & 0.04 & present study \\
\hline Hallaig Waterfall & Hal 39 & 5.10 & 0.758 & 0.10 & present study \\
\hline Hallaig Waterfall & Hal_39 & 5.10 & 0.585 & 0.02 & present study \\
\hline Hallaig Waterfall & Hal_43 & 5.08 & 0.711 & 0.04 & present study \\
\hline Hallaig Waterfall & Hal_43 & 5.08 & 0.690 & 0.03 & present study \\
\hline Robin Hood's Bay & HOOD 004 B & 5.05 & 0.569 & 0.18 & Korte and Hesselbo 2011 \\
\hline Robin Hood's Bay & HOOD 004 A & 5.05 & 0.592 & 0.14 & Korte and Hesselbo 2011 \\
\hline Hallaig Waterfall & Hal_60 & 4.87 & 0.832 & 0.09 & present study \\
\hline Hallaig Waterfall & Hal_60 & 4.87 & 0.735 & 0.07 & present study \\
\hline Hallaig Waterfall & Hal_71 & 4.80 & 0.707 & 0.22 & present study \\
\hline Hallaig Waterfall & Hal_71 & 4.80 & 0.675 & 0.17 & present study \\
\hline Hallaig Waterfall & Hal_73 & 4.80 & 0.632 & 0.07 & present study \\
\hline Hallaig Waterfall & Hal_73 & 4.80 & 0.615 & 0.07 & present study \\
\hline Ardnish & Ard_104 & 4.75 & 0.624 & 0.04 & present study \\
\hline Ardnish & Ard_104 & 4.75 & 0.617 & 0.07 & present study \\
\hline Ardnish & Ard_104 & 4.75 & 0.626 & 0.16 & present study \\
\hline Ardnish & Ard_105 & 4.75 & 0.595 & 0.09 & present study \\
\hline
\end{tabular}




\begin{tabular}{|c|c|c|c|c|c|}
\hline Ardnish & Ard_105 & 4.75 & 0.578 & 0.11 & present study \\
\hline Hallaig Waterfall & Hal_101 & 4.68 & 0.679 & 0.07 & present study \\
\hline Hallaig Waterfall & Hal_101 & 4.68 & 0.740 & 0.04 & present study \\
\hline Hallaig Waterfall & Hal_100 & 4.63 & 0.759 & 0.19 & present study \\
\hline Hallaig Waterfall & Hal_100 & 4.63 & 0.785 & 0.17 & present study \\
\hline Ardnish & Ard_102 & 4.58 & 0.645 & 0.11 & present study \\
\hline Ardnish & Ard_102 & 4.58 & 0.628 & 0.14 & present study \\
\hline Ardnish & Ard_102 & 4.58 & 0.719 & 0.37 & present study \\
\hline Ardnish & Ard_108 & 4.52 & 0.644 & 0.07 & present study \\
\hline Ardnish & Ard_108 & 4.52 & 0.682 & 0.19 & present study \\
\hline Ardnish & Ard_109 & 4.52 & 0.581 & 0.07 & present study \\
\hline Ardnish & Ard_109 & 4.52 & 0.611 & 0.14 & present study \\
\hline Ardnish & Ard_109 & 4.52 & 0.564 & 0.26 & present study \\
\hline Hallaig Waterfall & Hal_117 & 4.51 & 0.620 & 0.10 & present study \\
\hline Hallaig Waterfall & Hal_131 & 4.45 & 0.629 & 0.03 & present study \\
\hline Hallaig Waterfall & Hal_132 & 4.45 & 0.617 & 0.14 & present study \\
\hline Hallaig Waterfall & Hal_132 & 4.45 & 0.572 & 0.11 & present study \\
\hline Hallaig Waterfall & Hal_135 & 4.43 & 0.703 & 0.09 & present study \\
\hline Hallaig Waterfall & Hal_135 & 4.43 & 0.685 & 0.08 & present study \\
\hline Hallaig Waterfall & Hal_136 & 4.43 & 0.680 & 0.16 & present study \\
\hline Hallaig Waterfall & Hal_136 & 4.43 & 0.639 & 0.15 & present study \\
\hline Ardnish & Ard_127 & 4.31 & 0.565 & 0.10 & present study \\
\hline Ardnish & Ard_127 & 4.31 & 0.663 & 0.18 & present study \\
\hline Ardnish & Ard_127 & 4.31 & 0.625 & 0.23 & present study \\
\hline Hallaig Waterfall & Hal_158 & 4.30 & 0.605 & 0.06 & present study \\
\hline Hallaig Waterfall & Hal_158 & 4.30 & 0.655 & 0.02 & present study \\
\hline Ardnish & Ard_128 & 4.28 & 0.736 & 0.15 & present study \\
\hline Ardnish & Ard_128 & 4.28 & 0.628 & 0.22 & present study \\
\hline Ardnish & Ard_128 & 4.28 & 0.639 & 0.23 & present study \\
\hline Hallaig Waterfall & Hal_163 & 4.28 & 0.586 & 0.05 & present study \\
\hline Hallaig Waterfall & Hal_163 & 4.28 & 0.599 & 0.04 & present study \\
\hline Hallaig Waterfall & Hal_166 & 4.27 & 0.570 & 0.04 & present study \\
\hline Hallaig Waterfall & Hal_166 & 4.27 & 0.553 & 0.03 & present study \\
\hline Ardnish & Ard_122 & 4.26 & 0.601 & 0.05 & present study \\
\hline Ardnish & Ard_122 & 4.26 & 0.546 & 0.08 & present study \\
\hline Ardnish & Ard_122 & 4.26 & 0.741 & 0.30 & present study \\
\hline Ardnish & Ard_116 & 4.26 & 0.568 & 0.08 & present study \\
\hline Ardnish & Ard_116 & 4.26 & 0.579 & 0.13 & present study \\
\hline Ardnish & Ard_116 & 4.26 & 0.617 & 0.25 & present study \\
\hline Ardnish & Ard_117 & 4.26 & 0.639 & 0.03 & present study \\
\hline Ardnish & Ard_117 & 4.26 & 0.631 & 0.05 & present study \\
\hline Borreraig & Bor_144 & 4.26 & 0.677 & 0.14 & present study \\
\hline Hallaig Waterfall & Hal_168 & 4.26 & 0.684 & 0.03 & present study \\
\hline Hallaig Waterfall & Hal_168 & 4.26 & 0.696 & 0.02 & present study \\
\hline Ardnish & Ard_125 & 4.25 & 0.626 & 0.12 & present study \\
\hline Ardnish & Ard_125 & 4.25 & 0.610 & 0.12 & present study \\
\hline Ardnish & Ard_125 & 4.25 & 0.588 & 0.19 & present study \\
\hline Ardnish & Ard_120 & 4.25 & 0.603 & 0.13 & present study \\
\hline Ardnish & Ard_121 & 4.25 & 0.578 & 0.13 & present study \\
\hline
\end{tabular}




\begin{tabular}{|c|c|c|c|c|c|}
\hline Ardnish & Ard_120 & 4.25 & 0.557 & 0.14 & present study \\
\hline Ardnish & Ard_120 & 4.25 & 0.559 & 0.15 & present study \\
\hline Ardnish & Ard_121 & 4.25 & 0.617 & 0.18 & present study \\
\hline Ardnish & Ard_129 & 4.24 & 0.559 & 0.05 & present study \\
\hline Ardnish & Ard_129 & 4.24 & 0.639 & 0.16 & present study \\
\hline Ardnish & Ard_129 & 4.24 & 0.630 & 0.21 & present study \\
\hline Ardnish & Ard_133 & 4.24 & 0.575 & 0.07 & present study \\
\hline Ardnish & Ard_133 & 4.24 & 0.572 & 0.10 & present study \\
\hline Ardnish & Ard_133 & 4.24 & 0.580 & 0.11 & present study \\
\hline Ardnish & Ard_136 & 4.23 & 0.704 & 0.16 & present study \\
\hline Ardnish & Ard_136 & 4.23 & 0.786 & 0.21 & present study \\
\hline Borreraig & Bor_137 & 4.23 & 0.703 & 0.15 & present study \\
\hline Borreraig & Bor_137 & 4.23 & 0.656 & 0.10 & present study \\
\hline Hallaig Waterfall & Hal_176 & 4.22 & 0.644 & 0.02 & present study \\
\hline Hallaig Waterfall & Hal_176 & 4.22 & 0.685 & 0.01 & present study \\
\hline Hallaig Waterfall & Hal_177 & 4.22 & 0.626 & 0.21 & present study \\
\hline Hallaig Waterfall & Hal_177 & 4.22 & 0.570 & 0.18 & present study \\
\hline Ardnish & Ard_155 & 4.13 & 0.639 & 0.14 & present study \\
\hline Ardnish & Ard_155 & 4.13 & 0.669 & 0.39 & present study \\
\hline Ardnish & Ard_155 & 4.13 & 0.650 & 0.42 & present study \\
\hline Ardnish & Ard_151 & 4.07 & 0.637 & 0.07 & present study \\
\hline Ardnish & Ard_151 & 4.07 & 0.567 & 0.17 & present study \\
\hline Ardnish & Ard_148 & 4.07 & 0.655 & 0.11 & present study \\
\hline Ardnish & Ard_148 & 4.07 & 0.736 & 0.22 & present study \\
\hline Ardnish & Ard_146 & 4.06 & 0.619 & 0.13 & present study \\
\hline Ardnish & Ard_144 & 4.06 & 0.555 & 0.03 & present study \\
\hline Ardnish & Ard_144 & 4.06 & 0.545 & 0.05 & present study \\
\hline Ardnish & Ard_144 & 4.06 & 0.549 & 0.08 & present study \\
\hline Hallaig Waterfall & Hal_198 & 4.06 & 0.535 & 0.04 & present study \\
\hline Hallaig Waterfall & Hal_198 & 4.06 & 0.608 & 0.03 & present study \\
\hline Hallaig Waterfall & Hal_199 & 4.05 & 0.585 & 0.06 & present study \\
\hline Hallaig Waterfall & Hal_199 & 4.05 & 0.679 & 0.06 & present study \\
\hline Ardnish & Ard_142 & 4.05 & 0.582 & 0.08 & present study \\
\hline Ardnish & Ard_142 & 4.05 & 0.575 & 0.13 & present study \\
\hline Ardnish & Ard_142 & 4.05 & 0.650 & 0.28 & present study \\
\hline Lochaline & Alt M. 13A & 3.95 & 0.725 & 0.17 & present study \\
\hline Lochaline & Alt M. 12 & 3.90 & 0.846 & 0.45 & present study \\
\hline Lochaline & Alt M. 12 & 3.90 & 0.790 & 0.10 & present study \\
\hline Lochaline & Alt M. 12 & 3.90 & 0.792 & 0.09 & present study \\
\hline Lochaline & Alt M. 11 & 3.84 & 0.832 & 0.16 & present study \\
\hline Lochaline & Alt M. 10 & 3.79 & 0.679 & 0.10 & present study \\
\hline Lochaline & Alt M. 7 & 3.66 & 0.825 & 0.31 & present study \\
\hline Lochaline & Alt M. 4 & 3.66 & 0.821 & 0.17 & present study \\
\hline Lochaline & Alt M. 6 & 3.66 & 0.770 & 0.14 & present study \\
\hline Lochaline & Alt M. 9 & 3.66 & 0.676 & 0.08 & present study \\
\hline Lochaline & Alt M. 7 & 3.66 & 0.741 & 0.05 & present study \\
\hline Lochaline & Alt M. 7 & 3.66 & 0.782 & 0.05 & present study \\
\hline Lochaline & Alt M. 3 & 3.66 & 0.808 & 0.04 & present study \\
\hline Lochaline & Alt M. 8 & 3.66 & 0.808 & 0.04 & present study \\
\hline
\end{tabular}




\begin{tabular}{|c|c|c|c|c|c|}
\hline Lochaline & Alt L. 69 & 3.66 & 0.732 & 0.03 & present study \\
\hline Lochaline & Alt M. 5 & 3.66 & 0.781 & 0.03 & present study \\
\hline Lochaline & Alt M. 1 & 3.66 & 0.746 & 0.03 & present study \\
\hline Lochaline & Alt M. 3 & 3.66 & 0.707 & 0.02 & present study \\
\hline Lochaline & Alt L. 69 & 3.66 & 0.685 & 0.02 & present study \\
\hline Lochaline & Alt L. 69 & 3.66 & 0.654 & 0.01 & present study \\
\hline Lochaline & Alt L. 68 & 3.66 & 0.741 & 0.12 & present study \\
\hline Lochaline & Alt L. 67 & 3.66 & 0.723 & 0.03 & present study \\
\hline Lochaline & Alt L. 67 & 3.66 & 0.747 & 0.01 & present study \\
\hline Lochaline & Alt L. 66 & 3.66 & 0.675 & 0.12 & present study \\
\hline Lochaline & Alt L. 66 & 3.66 & 0.747 & 0.06 & present study \\
\hline Lochaline & Alt L. 65 & 3.63 & 0.798 & 0.36 & present study \\
\hline Lochaline & Alt L. 65 & 3.63 & 0.746 & 0.11 & present study \\
\hline Lochaline & Alt L. 65 & 3.63 & 0.736 & 0.09 & present study \\
\hline Lochaline & Alt L. 64 & 3.63 & 0.714 & 0.14 & present study \\
\hline Lochaline & Alt L. 64 & 3.63 & 0.728 & 0.09 & present study \\
\hline Lochaline & Alt L. 63 & 3.63 & 0.709 & 0.04 & present study \\
\hline Lochaline & Alt L. 62 & 3.62 & 0.752 & 0.10 & present study \\
\hline Lochaline & Alt L. 62 & 3.62 & 0.810 & 0.07 & present study \\
\hline Lochaline & Alt L. 61 & 3.60 & 0.810 & 0.23 & present study \\
\hline Lochaline & Alt L. 61 & 3.60 & 0.683 & 0.02 & present study \\
\hline Lochaline & Alt L. 61 & 3.60 & 0.701 & 0.02 & present study \\
\hline Lochaline & Alt L. 60 & 3.57 & 0.824 & 0.04 & present study \\
\hline Lochaline & Alt L. 60 & 3.57 & 0.735 & 0.03 & present study \\
\hline Lochaline & Alt L. 60 & 3.57 & 0.872 & 0.02 & present study \\
\hline Lochaline & Alt L. 60 & 3.57 & 0.847 & 0.01 & present study \\
\hline Lochaline & Alt L. 59 & 3.56 & 0.645 & 0.03 & present study \\
\hline Lochaline & Alt L. 59 & 3.56 & 0.694 & 0.00 & present study \\
\hline Lochaline & Alt L. 58 & 3.55 & 0.920 & 0.19 & present study \\
\hline Lochaline & Alt L. 58 & 3.55 & 0.768 & 0.12 & present study \\
\hline Lochaline & Alt L. 57 & 3.54 & 0.811 & 0.11 & present study \\
\hline Lochaline & Alt L. 56 & 3.54 & 0.742 & 0.06 & present study \\
\hline Lochaline & Alt L. 55 & 3.53 & 0.886 & 0.02 & present study \\
\hline Lochaline & Alt L. 54 & 3.52 & 0.820 & 0.18 & present study \\
\hline Lochaline & Alt L. 54 & 3.52 & 0.781 & 0.17 & present study \\
\hline Lochaline & Alt L. 54 & 3.52 & 0.775 & 0.14 & present study \\
\hline Lochaline & Alt L. 52 & 3.52 & 0.839 & 0.12 & present study \\
\hline Lochaline & Alt L. 49 & 3.49 & 0.721 & 0.03 & present study \\
\hline Lochaline & Alt L. 49 & 3.49 & 0.711 & 0.02 & present study \\
\hline Lochaline & Alt L. 47 & 3.46 & 0.807 & 0.28 & present study \\
\hline Lochaline & Alt L. 47 & 3.46 & 0.774 & 0.21 & present study \\
\hline Lochaline & Alt L. 46 & 3.46 & 0.788 & 0.15 & present study \\
\hline Lochaline & Alt L. 46 & 3.46 & 0.754 & 0.07 & present study \\
\hline Lochaline & Alt L. 45 & 3.46 & 0.683 & 0.07 & present study \\
\hline Lochaline & Alt L. 44 & 3.45 & 0.681 & 0.05 & present study \\
\hline Lochaline & Alt L. 44 & 3.45 & 0.740 & 0.05 & present study \\
\hline Lochaline & Alt L. 43 & 3.44 & 0.726 & 0.08 & present study \\
\hline Lochaline & Alt L. 42 & 3.43 & 0.862 & 0.19 & present study \\
\hline Lochaline & Alt L. 42 & 3.43 & 0.767 & 0.07 & present study \\
\hline
\end{tabular}




\begin{tabular}{|c|c|c|c|c|c|}
\hline Lochaline & Alt L. 41 & 3.42 & 0.833 & 0.21 & present study \\
\hline Lochaline & Alt L. 40 & 3.37 & 0.695 & 0.05 & present study \\
\hline Lochaline & Alt L. 40 & 3.37 & 0.710 & 0.04 & present study \\
\hline Lochaline & Alt L. 39 & 3.36 & 0.911 & 0.03 & present study \\
\hline Lochaline & Alt L. 39 & 3.36 & 0.950 & 0.03 & present study \\
\hline Lochaline & Alt L. 39 & 3.36 & 0.903 & 0.03 & present study \\
\hline Lochaline & Alt L. 38 & 3.34 & 0.857 & 0.02 & present study \\
\hline Lochaline & Alt L. 37 & 3.32 & 0.757 & 0.03 & present study \\
\hline Lochaline & Alt L. 37 & 3.32 & 0.752 & 0.03 & present study \\
\hline Lochaline & Alt L. 37 & 3.32 & 0.677 & 0.01 & present study \\
\hline Lochaline & Alt L. 36 & 3.30 & 0.711 & 0.03 & present study \\
\hline Lochaline & Alt L. 35 & 3.27 & 0.830 & 0.15 & present study \\
\hline Lochaline & Alt L. 34 & 3.23 & 0.879 & 0.02 & present study \\
\hline Lochaline & Alt L. 33 & 3.23 & 0.840 & 0.10 & present study \\
\hline Lochaline & Alt L. 33 & 3.23 & 0.802 & 0.08 & present study \\
\hline Lochaline & Alt L. 33 & 3.23 & 0.893 & 0.04 & present study \\
\hline Lochaline & Alt L. 33 & 3.23 & 0.886 & 0.03 & present study \\
\hline Lochaline & Alt L. 33 & 3.23 & 0.957 & 0.03 & present study \\
\hline Lochaline & Alt L. 31 & 3.17 & 0.804 & 0.11 & present study \\
\hline Lochaline & Alt L. 30 & 3.16 & 0.710 & 0.04 & present study \\
\hline Lochaline & Alt L. 30 & 3.16 & 0.907 & 0.01 & present study \\
\hline Lochaline & Alt S. 14 & 2.99 & 0.774 & 0.15 & present study \\
\hline Lochaline & Alt S. 14 & 2.99 & 0.704 & 0.10 & present study \\
\hline Lochaline & Alt S. 14 & 2.99 & 0.690 & 0.03 & present study \\
\hline Lochaline & Alt S. 13 & 2.98 & 0.905 & 0.05 & present study \\
\hline Lochaline & Alt S. 13 & 2.98 & 0.738 & 0.04 & present study \\
\hline Lochaline & Alt S. 13 & 2.98 & 0.723 & 0.03 & present study \\
\hline Lochaline & Alt S. 12 & 2.85 & 0.723 & 0.05 & present study \\
\hline Lochaline & Alt S. 12 & 2.85 & 0.741 & 0.03 & present study \\
\hline Lochaline & Alt S. 10 & 2.84 & 0.767 & 0.16 & present study \\
\hline Lochaline & Alt S. 11 & 2.84 & 0.995 & 0.22 & present study \\
\hline Lochaline & Alt S. 9 & 2.83 & 0.804 & 0.13 & present study \\
\hline Lochaline & Alt S. 8 & 2.81 & 0.768 & 0.05 & present study \\
\hline Lochaline & Alt S. 8 & 2.81 & 0.887 & 0.00 & present study \\
\hline Lochaline & Alt S. 6 & 2.79 & 0.756 & 0.06 & present study \\
\hline Lochaline & Alt S. 7 & 2.77 & 0.738 & 0.14 & present study \\
\hline Lochaline & Alt S. 7 & 2.77 & 0.704 & 0.11 & present study \\
\hline Lochaline & Alt S. 7 & 2.77 & 0.695 & 0.11 & present study \\
\hline Lochaline & Alt S. 5 & 2.77 & 0.739 & 0.04 & present study \\
\hline Lochaline & Alt S. 5 & 2.77 & 0.853 & 0.04 & present study \\
\hline Lochaline & Alt S. 4 & 2.76 & 0.673 & 0.03 & present study \\
\hline Lochaline & Alt S. 4 & 2.76 & 0.690 & 0.00 & present study \\
\hline Lochaline & Alt S. 3 & 2.75 & 0.744 & 0.09 & present study \\
\hline Lochaline & Alt S. 2 & 2.72 & 0.760 & 0.04 & present study \\
\hline Lochaline & Alt L. 28 & 2.72 & 0.768 & 0.11 & present study \\
\hline Lochaline & Alt S. 1 & 2.72 & 0.818 & 0.09 & present study \\
\hline Lochaline & Alt L. 27 & 2.71 & 0.655 & 0.02 & present study \\
\hline Lochaline & Alt L. 26 & 2.68 & 0.708 & 0.09 & present study \\
\hline Lochaline & Alt L. 25 & 2.68 & 0.754 & 0.06 & present study \\
\hline
\end{tabular}




\begin{tabular}{lccccc} 
Lochaline & Alt L. 24 & 2.60 & 0.696 & 0.00 & present study \\
Lochaline & Alt L. 24 & 2.60 & 0.767 & 0.00 & present study \\
Lochaline & Alt L. 21 & 2.54 & 0.735 & 0.05 & present study \\
Lochaline & Alt L. 21 & 2.54 & 0.743 & 0.03 & present study \\
Lochaline & Alt L. 20 & 2.53 & 0.750 & 0.12 & present study \\
Lochaline & Alt L. $17=15-1$; & 2.34 & 0.708 & 0.11 & present study \\
Lochaline & Alt L. $17=15-1$; & 2.34 & 0.788 & 0.08 & present study \\
Lochaline & Alt L. $17=15-1$; & 2.34 & 0.739 & 0.07 & present study \\
Lochaline & Alt L. 15 & 2.34 & 0.732 & 0.32 & present study \\
Lochaline & Alt L. 15 & 2.34 & 0.818 & 0.14 & present study \\
Lochaline & Alt L. 16 & 2.34 & 0.778 & 0.05 & present study \\
Lochaline & Alt L. 12 & 2.21 & 0.799 & 0.04 & present study \\
Lochaline & Alt L. 12 & 2.21 & 0.909 & 0.03 & present study \\
Lochaline & Alt L. 9 & 2.19 & 0.761 & 0.09 & present study \\
Lochaline & Alt L. 6 & 2.18 & 0.868 & 0.24 & present study \\
Lochaline & Alt L. 8 & 2.18 & 0.776 & 0.19 & present study \\
Lochaline & Alt L. 3 & 2.10 & 0.827 & 0.12 & present study \\
Lochaline & Alt L. 4 & 2.09 & 0.945 & 0.22 & present study \\
Lochaline & Alt L. 1 & 2.02 & 0.799 & 0.29 & present study \\
Lochaline & Alt L. 2 & 2.02 & 0.904 & 0.26 & present study \\
Lochaline & Alt L. 1 & 2.02 & 0.802 & 0.10 & present study \\
Lochaline & Alt L. 1 & 2.02 & 0.870 & 0.06 & present study \\
\hline
\end{tabular}


Calculations

\begin{tabular}{|c|c|c|c|c|c|c|c|c|c|c|c|c|c|c|c|c|c|c|c|c|c|}
\hline \multirow[t]{3}{*}{ Stage } & \multicolumn{3}{|c|}{ Ammonite Zone } & \multicolumn{4}{|c|}{ belemnites } & \multicolumn{4}{|c|}{ oysters } & \multirow[t]{3}{*}{ bel/biv } & \multirow[t]{3}{*}{ unc. } & \multicolumn{6}{|c|}{ reconstructed Seawater $\mathrm{Sr} / \mathrm{Ca}$} & \multicolumn{2}{|c|}{${ }^{87} \mathrm{Sr} /{ }^{86} \mathrm{Sr}$} \\
\hline & numerical & name & Age & $\mathrm{Sr} / \mathrm{Ca}$ & 2sd & 2err & $\mathbf{n}$ & $\mathrm{Sr} / \mathrm{Ca}$ & 2sd & 2err & $\mathbf{n}$ & & & \multicolumn{2}{|c|}{ belemnites } & \multicolumn{2}{|c|}{ oysters } & \multicolumn{2}{|c|}{ combined } & zone avg & 2err \\
\hline & J2002 & & G2004 & mmol/mol & & & & $\mathrm{mmol} / \mathbf{m o l}$ & & & & & & $\mathrm{Sr} / \mathrm{Ca}$ & 2err & $\mathrm{S} r / \mathbf{C a}$ & 2err & $\mathrm{Sr} / \mathrm{Ca}$ & 2err & & \\
\hline Callovian & 47.5 & lamberti & 163.81 & 1.21 & 0.02 & 0.02 & & & & & & & & 3.8 & 0.1 & & & 3.8 & 0.1 & 0.706852 & 0.000004 \\
\hline Callovian & 46.5 & athleta & 163.97 & 1.29 & 0.10 & 0.10 & & & & & & & & 4.1 & 0.3 & & & 4.1 & 0.3 & 0.706853 & 0.000004 \\
\hline Callovian & 45.5 & coronatum & 164.5 & 1.27 & 0.09 & 0.09 & & & & & & & & 4.0 & 0.3 & & & 4.0 & 0.3 & 0.706848 & 0.000012 \\
\hline Callovian & 44.5 & & 164.72 & 1.28 & 0.05 & 0.05 & & & & & & & & 4.1 & 0.2 & & & 4.1 & 0.2 & 0.706869 & 0.000008 \\
\hline Callovian & 42.5 & & 164.95 & 1.37 & 0.10 & 0.10 & & & & & & & & 4.4 & 0.3 & & & 4.4 & 0.3 & 0.706917 & 0.000012 \\
\hline Callovian & 41.5 & herveyi & 165.40 & 1.29 & 0.02 & 0.02 & & & & & & & & 4.1 & 0.1 & & & 4.1 & 0.1 & 0.706915 & 0.000005 \\
\hline Callovian & 38.5 & hodsoni & 166.07 & 1.50 & 0.04 & 0.04 & & & & & & & & 4.8 & 0.1 & & & 4.8 & 0.1 & 0.707013 & 0.000005 \\
\hline Bathonian & 37.5 & morrisi & 166.78 & 1.56 & 0.04 & 0.04 & & & & & & & & 4.9 & 0.1 & & & 4.9 & 0.1 & 0.707027 & 0.000003 \\
\hline Bathonian & 35.5 & progracilis & 167.37 & 1.73 & 0.19 & 0.19 & & & & & & & & 5.5 & 0.6 & & & 5.5 & 0.6 & 0.707033 & 0.000013 \\
\hline Bathonian & 34.5 & tenuiplicatus & 167.83 & 1.55 & 0.17 & 0.17 & & & & & & & & 4.9 & 0.5 & & & 4.9 & 0.5 & 0.707040 & 0.000008 \\
\hline Bathonian & 33.5 & zigzag & 168.28 & 1.47 & 0.08 & 0.08 & & & & & & & & 4.6 & 0.2 & & & 4.6 & 0.2 & 0.707043 & 0.000007 \\
\hline Bajocian & 32.5 & parkinsoni & 168.69 & 1.53 & 0.07 & 0.07 & & & & & & & & 4.9 & 0.2 & & & 4.9 & 0.2 & 0.707058 & 0.000008 \\
\hline Bajocian & 29.5 & humphriesianum & 169.7 & & & & & & & & & & & & & & & & & 0.707176 & 0.000003 \\
\hline Bajocian & 28.5 & propinquans & 169.9 & & & & & & & & & & & & & & & & & 0.707218 & 0.000013 \\
\hline Bajocian & 27.5 & laeviuscula & 170.1 & 1.66 & 0.35 & 0.10 & 12 & & & & & & & 5.3 & 0.3 & & & 5.3 & 0.3 & 0.707257 & 0.000013 \\
\hline Bajocian & 26.5 & ovalis & 170.2 & 1.43 & 0.22 & 0.05 & 18 & & & & & & & 4.5 & 0.2 & & & 4.5 & 0.2 & 0.707289 & 0.000010 \\
\hline Bajocian & 25.5 & discites & 170.3 & 1.62 & 0.44 & 0.10 & 20 & & & & & & & 5.1 & 0.3 & & & 5.1 & 0.3 & 0.707285 & 0.000007 \\
\hline Aalenian & 24.5 & concavum & 170.8 & & & & & & & & & & & & & & & & & 0.707288 & 0.000006 \\
\hline Aalenian & 23.5 & bradfordensis & 171.3 & 1.82 & 0.28 & 0.09 & 10 & & & & & & & 5.8 & 0.3 & & & 5.8 & 0.3 & 0.707277 & \\
\hline Aalenian & 22.5 & murchisonae & 172.1 & 1.55 & 0.31 & 0.10 & 10 & & & & & & & 4.9 & 0.3 & & & 4.9 & 0.3 & 0.707306 & 0.000015 \\
\hline Aalenian & 21.5 & scissum & 173.1 & 1.70 & 0.29 & 0.07 & 18 & & & & & & & 5.4 & 0.2 & & & 5.4 & 0.2 & 0.707309 & 0.000013 \\
\hline Aalenian & 20.5 & opalinum & 174.2 & 1.79 & 0.27 & 0.07 & 14 & & & & & & & 5.7 & 0.2 & & & 5.7 & 0.2 & 0.707296 & 0.000009 \\
\hline Toarcian & 19.5 & levesquei & 175.0 & 1.75 & 0.34 & 0.05 & 40 & & & & & & & 5.5 & 0.2 & & & 5.5 & 0.2 & 0.707294 & 0.000012 \\
\hline Toarcian & 18.5 & thouarense & 176.2 & & & & & & & & & & & & & & & & & 0.707267 & 0.000024 \\
\hline Toarcian & 17.5 & variabilis & 178.2 & 1.92 & 0.48 & 0.06 & 62 & & & & & & & 6.1 & 0.2 & & & 6.1 & 0.2 & 0.707259 & \\
\hline Toarcian & 16.5 & bifrons & 180.4 & 1.88 & 0.44 & 0.04 & 139 & & & & & & & 6.0 & 0.1 & & & 6.0 & 0.1 & 0.707237 & 0.000011 \\
\hline Toarcian & 15.5 & serpentinum & 181.7 & 1.76 & 0.35 & 0.04 & 80 & & & & & & & 5.6 & 0.1 & & & 5.6 & 0.1 & 0.707162 & 0.000027 \\
\hline Toarcian & 14.5 & tenuicostatum & 182.7 & 1.34 & 0.36 & 0.06 & 36 & & & & & & & 4.2 & 0.2 & & & 4.2 & 0.2 & 0.707097 & 0.000008 \\
\hline Pliensbachian & 13.5 & spinatum & 184.6 & 1.63 & 0.58 & 0.15 & 15 & 0.47 & 0.06 & 0.04 & 3 & 3.5 & 0.6 & 5.2 & 0.5 & 4.7 & 0.4 & 4.9 & 0.7 & 0.707099 & 0.000011 \\
\hline Pliensbachian & 12.5 & margaritatus & 186.5 & 1.64 & 0.44 & 0.08 & 29 & 0.50 & 0.23 & 0.06 & 15 & 3.3 & 0.6 & 5.2 & 0.3 & 5.0 & 0.6 & 5.1 & 0.3 & 0.707175 & 0.000012 \\
\hline Pliensbachian & 11.5 & davoei & 188.5 & 1.49 & 0.71 & 0.25 & 8 & 0.50 & 0.16 & 0.03 & 24 & 3.0 & 0.7 & 4.7 & 0.8 & 5.0 & 0.3 & 4.8 & 0.3 & 0.707232 & 0.000011 \\
\hline Pliensbachian & 10.5 & ibex & 189.4 & 1.37 & 0.75 & 0.13 & 32 & 0.45 & 0.16 & 0.04 & 14 & 3.0 & 0.6 & 4.3 & 0.4 & 4.5 & 0.4 & 4.4 & 0.2 & 0.707272 & 0.000014 \\
\hline Pliensbachian & 9.5 & jamesoni & 190.8 & 1.44 & 0.61 & 0.11 & 28 & 0.50 & 0.17 & 0.02 & 52 & 2.8 & 0.4 & 4.6 & 0.4 & 5.0 & 0.2 & 4.8 & 0.7 & 0.707344 & 0.000010 \\
\hline Sinemurian & 8.5 & raricostatum & 192.8 & 1.77 & 0.43 & 0.12 & 13 & 0.58 & 0.11 & 0.02 & 29 & 3.1 & 0.3 & 5.6 & 0.4 & 5.8 & 0.2 & 5.7 & 0.2 & 0.707456 & 0.000020 \\
\hline Sinemurian & 7.5 & oxynotum & 193.8 & 1.59 & 0.17 & 0.12 & 2 & 0.55 & 0.22 & 0.08 & 7 & 2.9 & 0.7 & 5.0 & 0.4 & 5.5 & 0.8 & 5.3 & 0.7 & 0.707571 & \\
\hline Sinemurian & 6.5 & obtusum & 195.3 & 1.82 & 0.45 & 0.20 & 5 & 0.55 & 0.10 & 0.06 & 3 & 3.3 & 0.7 & 5.8 & 0.6 & 5.5 & 0.6 & 5.6 & 0.4 & 0.707528 & 0.000019 \\
\hline Sinemurian & 5.5 & turneri & 196.3 & 2.29 & 0.40 & 0.28 & 2 & 0.66 & 0.14 & 0.02 & 36 & 3.5 & 0.6 & 7.2 & 0.9 & 6.6 & 0.2 & 6.9 & 1.0 & 0.707574 & 0.000037 \\
\hline Sinemurian & 4.5 & semicostatum & 197.8 & & & & & 0.63 & 0.12 & 0.01 & 94 & & & & & 6.3 & 0.1 & 6.3 & 0.1 & 0.707654 & 0.000074 \\
\hline Sinemurian & 3.5 & bucklandi & 199.3 & & & & & 0.78 & 0.15 & 0.02 & 84 & & & & & 7.8 & 0.2 & 7.8 & 0.2 & 0.707747 & 0.000030 \\
\hline Hettangian & 2.5 & angulata & 200.1 & & & & & 0.78 & 0.15 & 0.02 & 50 & & & & & 7.8 & 0.2 & 7.8 & 0.2 & 0.707757 & 0.000008 \\
\hline Hettangian & 1.5 & liasicus & 200.9 & & & & & & & & & & & & & & & & & 0.707756 & 0.000021 \\
\hline Hettangian & 0.5 & planorbis & 201.1 & & & & & & & & & & & & & & & & & 0.707775 & 0.000036 \\
\hline
\end{tabular}

